\title{
Aerodynamic and Acoustic Flight Test Results for the Stratospheric Observatory for Infrared Astronomy
}

\author{
Stephen B. Cumming, ${ }^{1}$ Larry J. Cliatt, II, ${ }^{2}$ Michael A. Frederick, ${ }^{3}$ and Mark S. Smith ${ }^{4}$ \\ NASA Dryden Flight Research Center, Edwards, CA, 93523
}

\begin{abstract}
As part of the Stratospheric Observatory for Infrared Astronomy program, a 747SP airplane was modified to carry a $2.5-\mathrm{m}$ telescope in the aft section of the fuselage. The resulting airborne observatory allows for observations above 99 percent of the water vapor in the atmosphere. The open cavity created by the modifications had the potential to significantly affect the airplane in the areas of aerodynamics and acoustics. Several series of flight tests were conducted to clear the operating envelope of the airplane for astronomical observations, planned to be performed between the altitudes of $35,000 \mathrm{ft}$ and $45,000 \mathrm{ft}$. The flight tests were successfully completed. Cavity acoustics were below design limits, and the overall acoustic characteristics of the cavity were better than expected. The modification did have some effects on the stability and control of the airplane, but these effects were not significant. Airplane air data systems were not affected by the modifications. This paper describes the methods used to examine the aerodynamics and acoustic data from the flight tests and provides a discussion of the flight-test results in the areas of cavity acoustics, stability and control, and air data.
\end{abstract}

\section{Nomenclature}

$\begin{array}{ll}\text { AA } & =\text { aperture assembly } \\ \text { CDS } & =\text { cavity door system } \\ \text { CFD } & =\text { computational fluid dynamics } \\ \text { CPT } & =\text { cable position transducer } \\ C_{m_{\alpha}} & =\text { pitching moment coefficient due to angle of attack, deg }{ }^{-1} \\ C_{m_{\tilde{e}}} & =\text { pitching moment coefficient due to elevators, deg }{ }^{-1} \\ C_{n_{\beta}} & =\text { yawing moment coefficient due to angle of sideslip, deg }{ }^{-1} \\ C_{n_{\delta ்}} & =\text { yawing moment coefficient due to rudder, deg }{ }^{-1} \\ \text { DLR } & =\text { Deutsches Zentrum fur Luft- und Raumfahrt (Cologne, Germany) } \\ \text { EGI } & =\text { embedded GPS/INS } \\ \text { FADS } & =\text { flush air data sensing } \\ \text { FFT } & =\text { fast Fourier transform } \\ \text { GPS } & =\text { global positioning system } \\ \text { INS } & =\text { inertial navigation system } \\ \text { KAO } & =\text { Kuiper airborne observatory } \\ \text { LFD } & =\text { lower flexible door } \\ n & =\text { number of measurements in sample window } \\ p & =\text { pressure, psi } \\ \text { PSD } & =\text { power spectral density } \\ \text { PUPO } & =\text { pull up, push over (maneuver) }\end{array}$

\footnotetext{
${ }_{1}^{1}$ Aerospace Engineer, Aerodynamics and Propulsion Branch, P.O. Box 273/ MS 2228, AIAA Senior Member.

${ }^{2}$ Aerospace Engineer, Aerodynamics and Propulsion Branch, P.O. Box 273/ MS 2228, AIAA nonmember.

${ }^{3}$ Aerospace Engineer, Aerodynamics and Propulsion Branch, P.O. Box 273/ MS 2228, AIAA Senior Member.

${ }^{4}$ Aerospace Engineer, Aerodynamics and Propulsion Branch, P.O. Box 273/ MS 2228, AIAA nonmember.
} 


$\begin{array}{ll}\bar{q} & =\text { dynamic pressure, psf } \\ \text { RVSM } & =\text { reduced vertical separation minimum } \\ \text { SOFIA } & =\text { Stratospheric Observatory for Infrared Astronomy } \\ \text { SPL } & =\text { sound pressure level } \\ \text { TA } & =\text { telescope assembly } \\ \mathrm{URD} & =\text { upper rigid door } \\ \alpha & =\text { angle of attack, deg } \\ \beta & =\text { angle of sideslip, deg } \\ \text { Subscripts } & \\ \text { AC } & =\text { oscillatory with respect to the moving average } \\ \text { avg } & =\text { average over sample window } \\ \text { max } & =\text { theoretical maximum } \\ \text { ref } & =\text { reference } \\ \text { RMS } & =\text { root mean squared }\end{array}$

\section{Introduction}

$\mathrm{T}$ The Stratospheric Observatory for Infrared Astronomy (SOFIA) is an airborne astronomical observatory developed through a partnership between the National Aeronautics and Space Administration (NASA) and Deutsches Zentrum fur Luft- und Raumfahrt (DLR) (Cologne, Germany). The SOFIA airplane is a modified 747SP airplane (The Boeing Company, Chicago, Illinois) carrying an optical/infrared/sub-millimeter reflecting telescope with an effective diameter of $2.5 \mathrm{~m}$. The observatory was designed to perform observations between the altitudes of $35,000 \mathrm{ft}$ and 45,000 ft, where it is above 99 percent of the water vapor in the atmosphere. The SOFIA program follows other NASA airborne observatories, most recently the Gerard P. Kuiper Airborne Observatory (KAO). ${ }^{1}$

The airplane was modified for use as an observatory by installing the telescope and associated equipment in the aft section of the airplane. To provide maximum optical performance, an open-air cavity was created and a door system was installed. The door system allows for an unvignetted elevation range of 23 deg to 59 deg. An unvignetted image is one without any distortions at the periphery of the image. For the SOFIA program, an unvignetted image was generally considered to be one unobstructed by any portion of the structure around the cavity door. A passive flow control system was designed and incorporated into the cavity and door design to minimize acoustic resonance in the cavity. ${ }^{2,3}$ Requirements for the SOFIA program dictated an operational lifetime in excess of 20 years, with 960 hours of observations per year for the observatory. Each research flight should provide the capability for $6 \mathrm{hr}$ of observations at an altitude of at least $41,000 \mathrm{ft}$.

To ensure the airworthiness of the airplane, and the observatory as a whole, for this mission a substantial flight envelope expansion process was necessary. The structural integrity of the modified airplane had to be verified. The acoustic environment inside the cavity had to be characterized to ensure that acoustic levels remained below design limits, both to prevent structural fatigue and to allow the observatory to meet image quality requirements. The stability and controllability of the modified airplane also had to be evaluated, both in closed-door and open-door configurations. The calibrations of the production pitot-static system had to be verified, and the research air data system had to be calibrated. In addition, all of the new systems installed to support the telescope and the observatory had to be tested, and their functionality verified. Testing began in April 2007; the airplane was cleared for science operations after approximately four years. This paper concentrates on the cavity acoustics, stability and control, and air data portions of the flight-testing.

\section{Vehicle Description}

The SOFIA airplane is a modified Boeing 747SP airplane, serial number 21441, line number 306. The airplane was initially delivered to Pan Am Airlines (New York, New York) in 1977 and later transferred to United Airlines (Chicago, Illinois) in 1986. The airframe was acquired by NASA in 1997. The maximum take-off weight is $696,000 \mathrm{lb}$, and the maximum altitude is $45,100 \mathrm{ft}$. The airplane is equipped with four Pratt \& Whitney (East Hartford, Connecticut) JT9D-7J engines, each capable of producing approximately 50,000 lb of static thrust. Primary control surfaces consist of elevators for pitch control, ailerons and spoilers for roll control, and a split rudder for yaw control. In addition, the horizontal stabilizer can be moved for pitch trim. The airplane prior to the SOFIA modifications is shown in Fig. 1. 


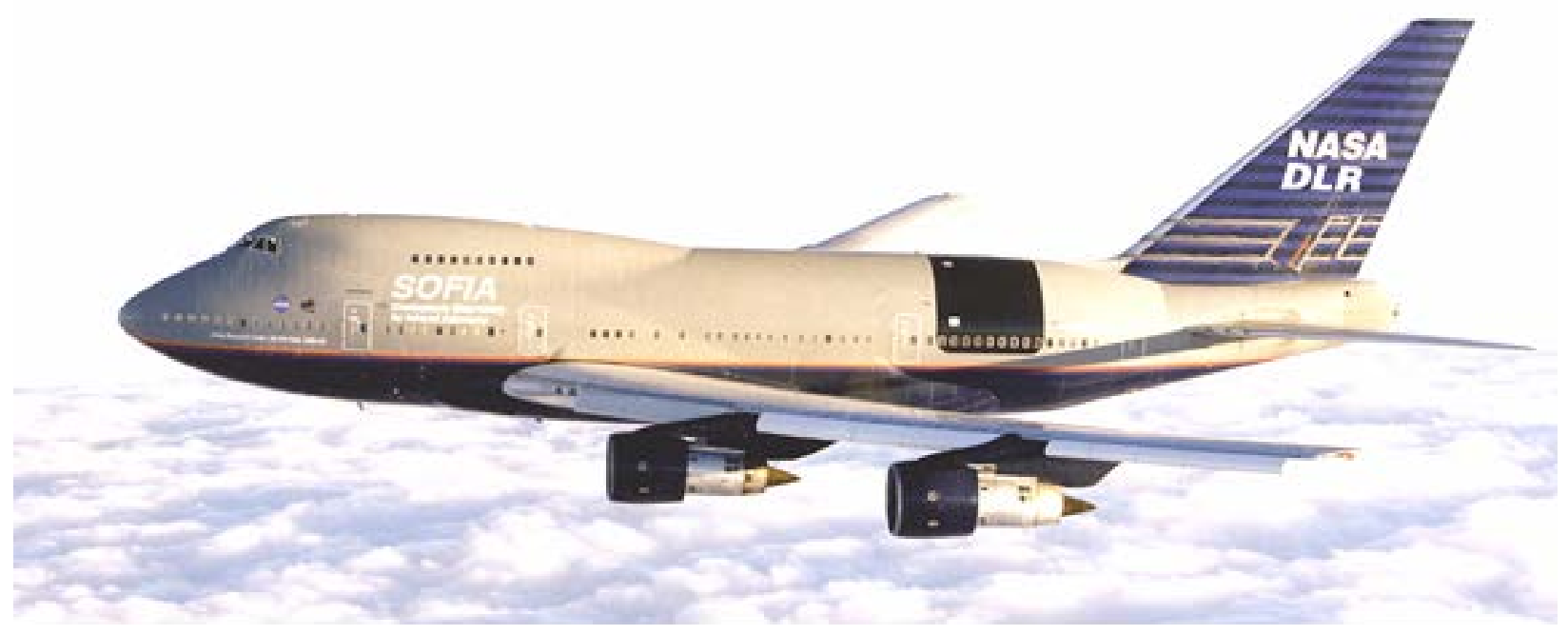

Figure 1. The SOFIA 747SP airplane prior to modification.

To support the stated mission as an airborne observatory, the SOFIA airplane was extensively modified. The bulk of these modifications were performed by L3 Communications (New York, New York) at their Integrated Systems facility in Waco, Texas. A cavity area for the telescope assembly (TA) was created by the installation of two new bulkheads. The forward bulkhead serves as the aft pressure barrier for the cabin and as the mechanical interface to which the TA is mounted. The aft bulkhead was installed to provide a finite cavity volume and a more sterile environment for the TA. The TA and portions of both bulkheads can be seen in Fig. 2. An integrated passive flow control system and cavity door system (CDS) was developed through wind-tunnel testing. ${ }^{3,4}$ The SOFIA cavity door system consists of a forward fairing, an aft fairing, an upper rigid door (URD), a lower flexible door (LFD), and an aperture assembly (AA). This system can be seen in Fig. 3 in an open configuration. When closed, the URD covers the aperture and seals the cavity. The LFD provides a surface below the aperture, between the lower AA surface and the fuselage, as the elevation angle increases. The AA consists of sidewalls on the forward, upper, and lower sides of the aperture, and a ramp at the aft. The ramp serves as the primary means of flow control, minimizing shear layer ingestion into the cavity. In a nominal system configuration for astronomical observations, the URD moves to expose the aperture and the cavity, and the URD, the AA, and the LFD all rotate together, tracking the rotation of the TA. The TA was designed with three degrees of freedom, including elevation, cross-elevation, and line-of-sight. Figure 4 shows the SOFIA airplane flying in an open-door configuration. 


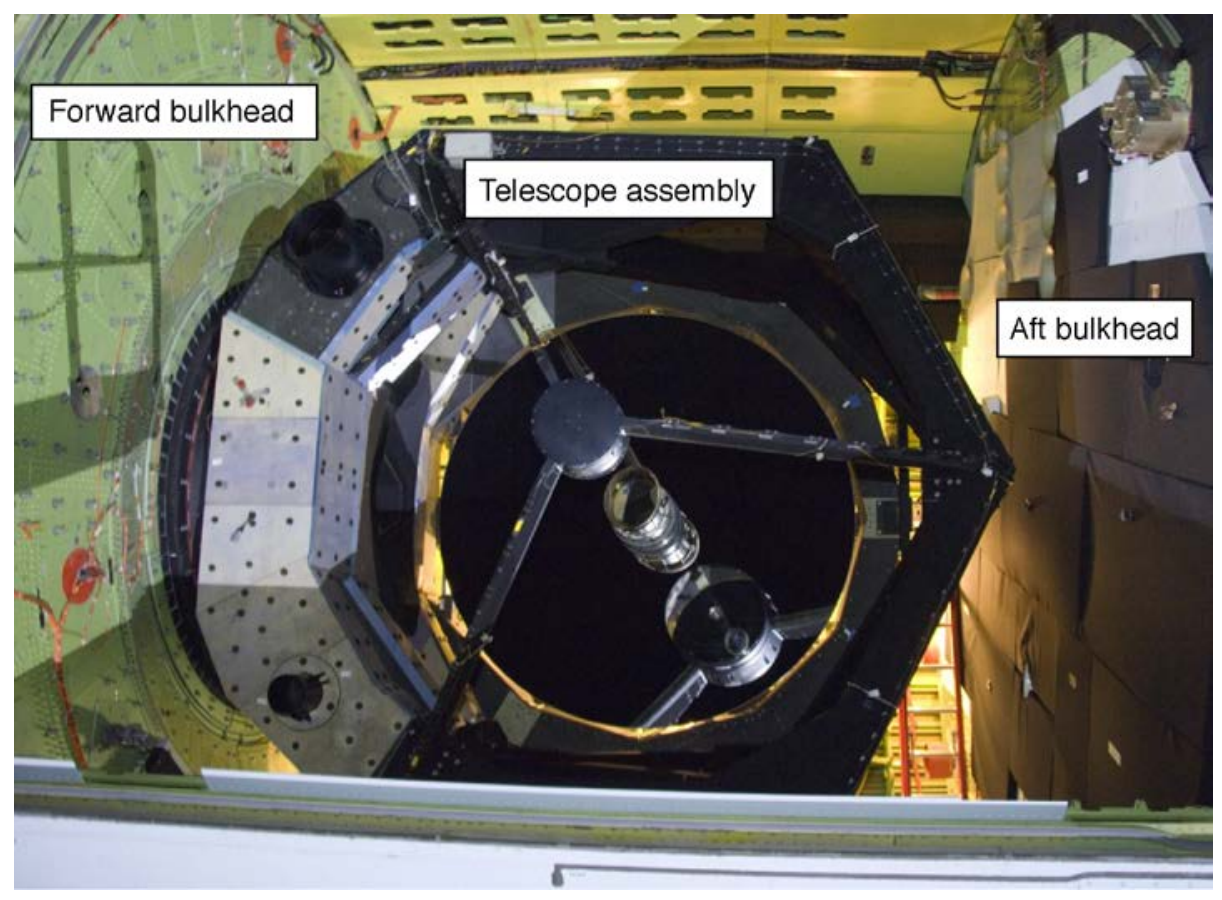

Figure 2. View of the telescope assembly and cavity.

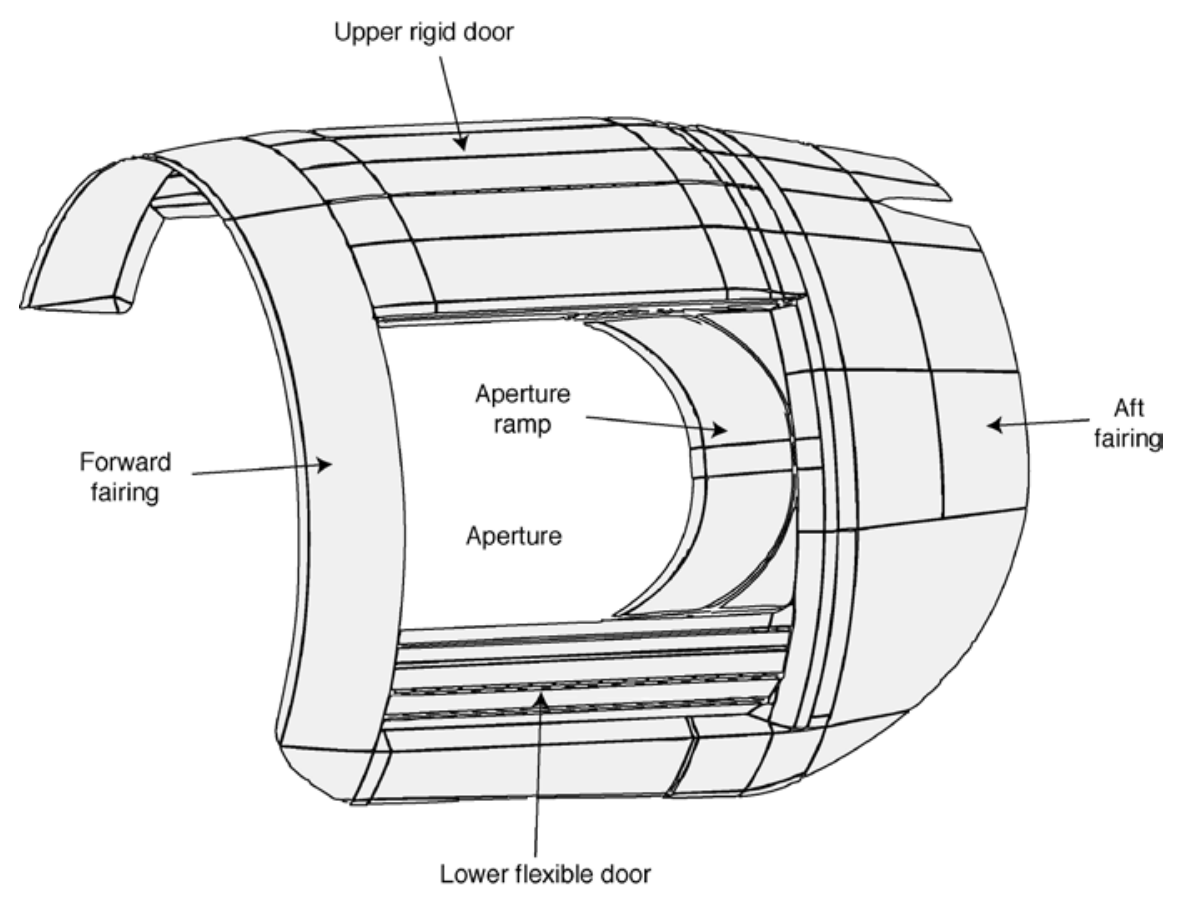

Figure 3. SOFIA cavity door system.

American Institute of Aeronautics and Astronautics 




Figure 4. The SOFIA airplane flying in an open-door configuration.

In addition to the structural modifications, substantial flight-test instrumentation was added to the SOFIA airplane to support safety monitoring and the data collection envelope expansion flights. Cable position transducers (CPTs), or string potentiometers, synchros, and discrete switches were installed to measure control surface positions. Flush pressure ports were installed in the nose to provide data for angle of attack and angle of sideslip. The pitot-static system of the airplane was instrumented to provide static- and total-pressure readings. A research total air temperature probe was installed to provide accurate total air temperature measurements. An embedded GPS/INS (EGI) unit was installed to provide airplane angular rates, linear accelerations, and Euler angles. A differential GPS unit was also installed to provide accurate altitude and ground speed values. The walls of the TA cavity, the URD, the LFD, and the AA were all instrumented with a total of 226 pressure sensors, to measure both steady-state and unsteady pressures. Standard 747SP airplane systems instrumentation, including fuel tank quantities and engine parameters were also available. Strain gages and accelerometers were also installed throughout the airplane structure to support structural integrity during ground and flight tests, and flutter flight tests.

\section{Preliminary Analyses and Tests}

Prior to the modifications to the SOFIA airplane and the flight-testing, substantial analyses and tests were performed both to design the modifications and to develop predictions for flight test, including predictions related to structural responses, stability and control, and acoustics. From an aerodynamics and acoustics standpoint, there are three major tests of interest: a series of five 7-percent-scale wind-tunnel tests performed to design the cavity; a series of three 3-percent-scale wind-tunnel tests that investigated the effects of the cavity on airplane stability and controllability; and a series of baseline flights with the airplane prior to the SOFIA modifications. In addition to these three tests, substantial computational fluid dynamics (CFD) analyses were performed, focusing on the flow in and around the cavity as well as the effects of the cavity on the airplane. ${ }^{5,6}$

A series of five 7-percent-scale wind-tunnel tests were performed in the 14-ft Transonic Wind Tunnel at the NASA Ames Research Center (Sunnyvale, California), ${ }^{4}$ beginning in 1990 and ending in 1997. The tests were performed to serve as design and validation tests for the SOFIA program cavity design. Mach numbers for these tests ranged from 0.30 to 0.92 . The design was finalized after the fifth wind-tunnel entry. The final design was referred to as the "partial external door configuration." The partial external door configuration developed from these tests is shown in Fig. 5. Throughout all of the 7-percent-scale wind-tunnel tests, pressure data were captured in and around the cavity. These data were used primarily to capture cavity acoustics inside the cavity and to examine flow effects just downstream of the cavity. 


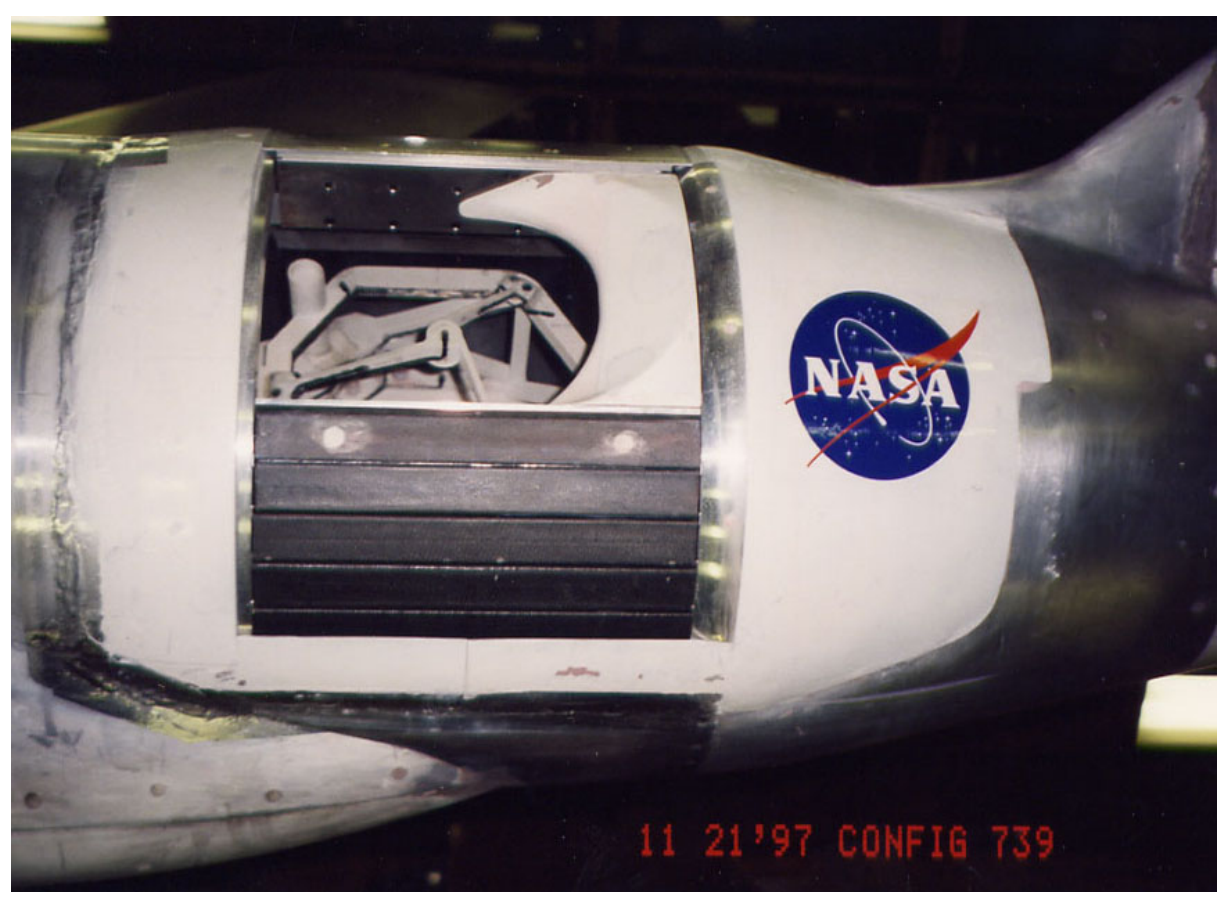

Figure 5. Partial external door configuration wind-tunnel model.

Acoustic data from the 7-percent-scale wind-tunnel tests were scaled to flight conditions following the wind-tunnel testing. To scale the frequency of the pressure fluctuations, the ratio of the speed of sound divided by the characteristic length of the wind tunnel to flight was used. This translates to the scale factor of the model multiplied by the ratio of the speed of sound in the wind tunnel to the speed of sound in flight. To scale the amplitude of the pressure fluctuations, they were multiplied by the ratio of dynamic pressure in flight to dynamic pressure in the wind tunnel. The pressure sensors used in these tests had a bandwidth of $1000 \mathrm{~Hz}$.

A series of three 3-percent-scale wind-tunnel tests were performed at two facilities: the University of Washington (Seattle, Washington) Aeronautical Laboratory Kirsten Wind Tunnel (UWAL) and the Boeing Transonic Wind Tunnel (BTWT). These tests began in 1998 and were completed in 1999. The UWAL entry concentrated on low-speed flight conditions and was performed at Mach 0.245 . The tests examined various flap settings and landing gear configurations. The BTWT entry covered a range of higher-speed flight conditions, from Mach 0.40 to Mach 0.975 . The tests were performed primarily to compile stability and control data for the modified SOFIA airplane. In addition to the stability and control data collected, steady-state pressures along the fuselage and acoustics inside the cavity were also collected (SOFIA document 05-N-19980930-001, "Synopsis of Low-Speed Wind Tunnel Test," dated September 30, 1998, and SOFIA document 05-N-19981118-001, "Synopsis of High-Speed Wind Tunnel Test,” dated November 18, 1998).

Prior to the SOFIA modifications, a series of baseline flights were performed with the 747SP airplane. The flight tests were performed by Kohlman Systems Research (Lawrence, Kansas). A total of 17 baseline flights were flown. The intent of these flights was to gather baseline data to support future flight-testing with the modified airplane and to provide a baseline for Federal Aviation Administration (FAA) certification. The suite of instrumentation for these flights was similar to the instrumentation installed for the SOFIA envelope expansion flights, but without the additional sensors required due to the modifications. Baseline stability and control, handling qualities, and performance data were all collected. In addition, investigations into stall speeds, flutter, and airplane strains were all completed. As part of these flights, initial flow angle calibrations were created for a flush air data sensing (FADS) system that was installed to provide flow angle measurements. The FADS system used in this flight series was a four-port configuration. Parameter estimation maneuvers from the baseline flights were later analyzed at the NASA Dryden Flight Research Center (Edwards, California) to provide stability and control data to which the results for the modified aircraft could be compared. 


\section{Flight-Test Approach}

Substantial flight-testing of the SOFIA airplane was performed following the completion of the observatory modifications. This testing was required to clear the airplane for science operation and allow for an observatory capable of meeting the planned operational lifespan of 20 years. The flight tests were designed to support NASA public-use certification for the airplane instead of the originally planned FAA certification. The NASA public-use designation was judged to be more appropriate for the airplane.

\section{A. Flight-Test Phases}

Envelope expansion flight-testing of the SOFIA airplane was broken up into three phases, or segments. Each flight segment had unique objectives. The first flight segment, designated Segment 0, consisted of closed-door envelope clearance for the ferry flight from Waco, Texas to the NASA Dryden Flight Research Center. The second segment, designated Segment 1, consisted of closed-door envelope expansion and collection of baseline closed-door data. The third segment, Segment 2, consisted of open-door envelope expansion and collection of data to clear the airplane for operational open-door flights. The closed-door and open-door flight envelopes that were cleared during Segments 1 and 2 are depicted in Fig. 6.

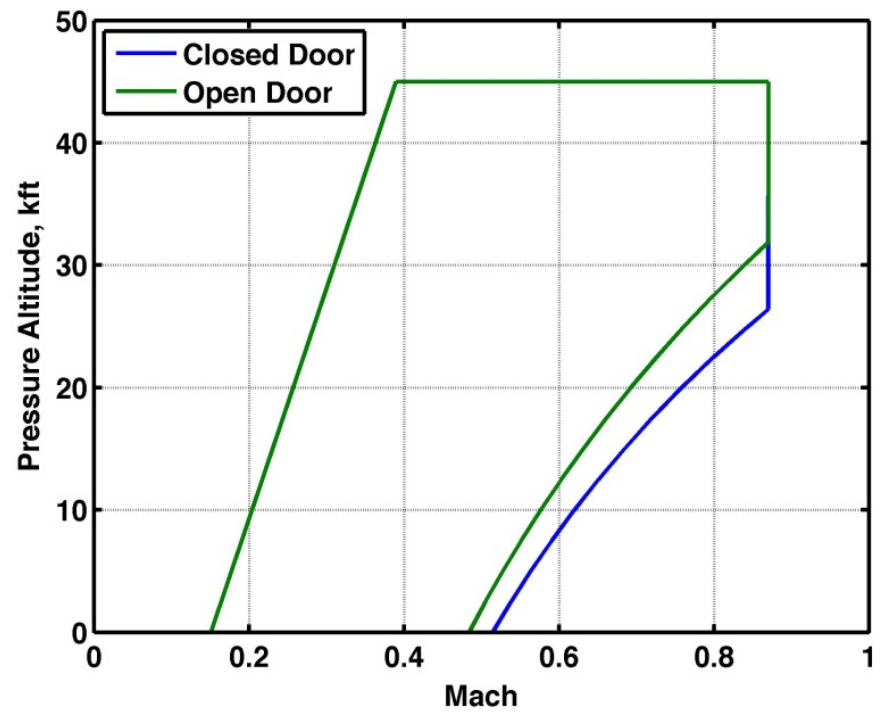

Figure 6. SOFIA closed-door and open-door flight envelopes.

Segment 0 was the initial set of test flights of the SOFIA 747SP airplane following the various modifications completed to transform the airplane into a flying observatory. This segment consisted of four flights, including a ferry flight. These flights cleared a flight envelope sufficient to ferry the airplane from Waco, Texas to the NASA Dryden Flight Research Center. Controllability, air data systems, and structural dynamics were all areas of interest during these flights, but the primary area of interest was airplane structural integrity after the modification. During this segment of flights, the FADS system was flown in the four-port configuration that had been calibrated during the baseline flights. Three envelope expansion flights were flown at Waco; flight-test maneuvers were performed and data were collected. Following the completion of the envelope expansion flights, the airplane was successfully ferried to the NASA Dryden Flight Research Center.

Segment 1 flights were flown to clear the operational closed-door envelope for the SOFIA airplane. This segment consisted of six flights. The primary objective of this flight series was to evaluate the structural integrity of the airplane over the full flight envelope. Other objectives included calibration of a five-port FADS and production air data systems and generating closed-door baseline data for airplane stability and control. Preliminary tests of TA functionality were also performed.

Segment 2 was the final envelope expansion segment, consisting of 25 flights flown primarily for open-door envelope expansion. The primary objective during these flights was to characterize the aero-acoustic behavior of the open cavity. The airplane had to be cleared over the operational flight envelope for each unique cavity configuration, which included AA elevation, TA elevation, and URD position. In order to both provide a range of AA/TA aligned 
elevations, and to minimize the amount of flight-testing required, the following AA/TA aligned elevations were flight-tested: $23^{\circ}, 30^{\circ}, 40^{\circ}, 50^{\circ}$, and $57^{\circ}$. In addition, the following URD positions would be tested: 10-percent open, 40-percent open, 70-percent open and 100-percent open. For AA/TA elevations other than $23^{\circ}$, CDS systems prevented 100-percent open testing, so these test points were performed at the 95-percent open URD position. Telescope assembly/ aperture assembly misalignment testing was also performed to fully clear operational configurations. Telescope assembly/ aperture assembly misalignment testing consisted of moving the TA through its elevation range with the aperture at the following fixed elevations: $23^{\circ}, 40^{\circ}$, and $57^{\circ}$; and moving the $\mathrm{TA}+/-2.5^{\circ}$ in cross elevation.

Other objectives for Segment 2 included investigating possible effects on structural dynamics, cavity acoustics, stability and control, and air data systems, and collecting other data necessary to qualify the airplane to fly operationally for at least 20 years. This included collecting the required data to certify the airplane for reduced vertical separation minimum (RVSM) operations. An additional port was added to the FADS system to provide increased reliability for the system, requiring additional calibration data. The other data collected included information on structural integrity and reliability of newly installed or modified airplane systems, including various TA systems.

This paper discusses Segments 0, 1, and 2, with emphasis on Segment 2 flight tests and results.

\section{B. Flight-Test Concerns}

There were several areas of concern during the various envelope expansion phases of the SOFIA project. These areas included structural integrity, structural dynamics, cavity acoustics, stability and control, and air data systems. This paper discusses the areas of acoustics, stability and control, and air data systems.

There were no substantial aerodynamics-related concerns associated with Segments 0 and 1, but it was still important to verify that the modifications to the outer mold line of the airplane, that is, the fairings and URD, did not affect flow over the empennage significantly enough to affect stability or controllability. A scenario in which these effects were significant was not deemed likely. In addition, the five-port FADS system and the instrumented pitot-static system needed calibration to support future flight tests.

One major factor that was considered during the flight-test planning and the flight tests themselves was the reliability of the CDS. This system was designed to avoid damage to the aircraft structure. As such, the system had multiple potential failure modes in which the door would fail in the open position. This meant that at any time during Segment 2 envelope expansion, the airplane needed to be cleared to return to base and land in the configuration under test, or at any URD position at that AA/TA elevation angle. During the KAO program, one of the most alarming acoustic resonances that was encountered occurred during an open-door landing, thus, acoustics in this situation were of particular interest. In order to clear the airplane for operations, open-door landings were planned during Segment 2 envelope expansion flights.

\section{Cavity Acoustics Concerns}

A primary area of concern during open-door flight-testing was the existence of substantial acoustic energy concentrated over a narrow frequency range at one or more flight conditions. In particular, the possibility of cavity resonance was a major concern. A cavity acoustic resonance event could result in significant structural damage to either the airplane structure or the TA. The extent and location of the damage would depend on the specifics of the acoustic event, including the frequency and spatial coherence of the resonance. Structural damage could be severe if the acoustic frequencies were at, or close to, the natural frequencies of the structure of the airplane. The acoustics coupling with the structural modes of the cavity were identified as an area of major concern, both by the project and independent review teams. ${ }^{3}$ Even if a severe resonance were not encountered, significant cavity acoustics could substantially degrade the pointing performance of the telescope. Based on results from the various wind-tunnel tests and CFD analyses, a maximum acoustic environment was defined for the telescope and associated systems. All systems were required to function within specifications for that environment. The environment is defined per the power spectral density (PSD) shown in Fig. 7. The PSD was considered to be the maximum reasonable cavity acoustic level expected in flight after completion of the 7-percent- and 3-percent-scale wind-tunnel tests, and was used in the design of the TA components. Cavity structures were generally designed to withstand a sound pressure level (SPL) of $146 \mathrm{~dB}$ with all of the energy concentrated at the natural frequency of that structure. Since the natural frequencies of the cavity structure and the TA components were below $200 \mathrm{~Hz}$, frequencies above that level were not generally considered significant. 

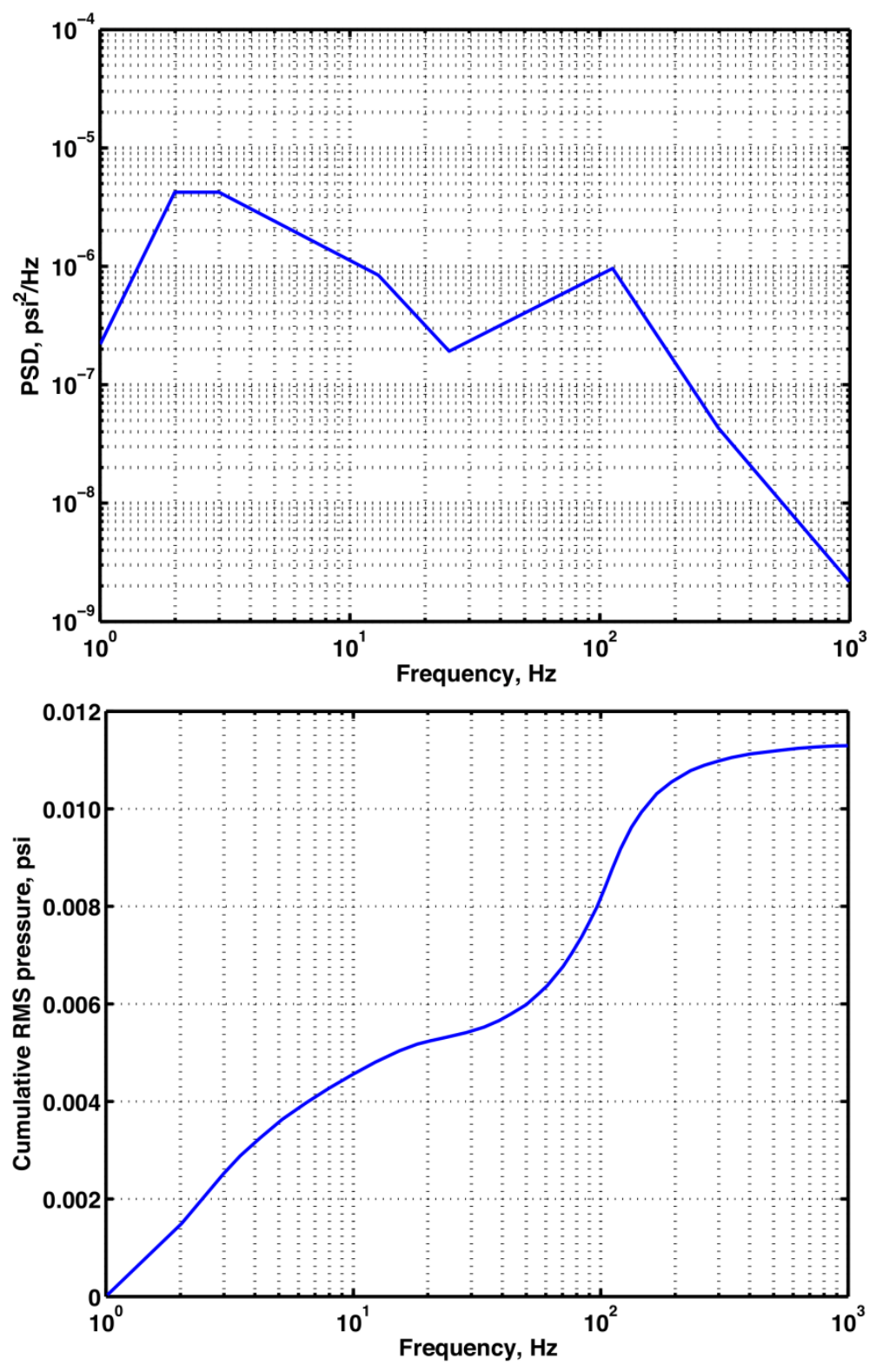

Figure 7. Maximum cavity acoustic environment for telescope assembly component design.

The 7-percent-scale wind-tunnel tests predicted an SPL inside the cavity of approximately $125 \mathrm{~dB}$ at the design point, an altitude of $41,000 \mathrm{ft}$ and Mach 0.85 , with no substantial energy at any particular frequency. The results from these tests indicated that the configuration was a robust design, with good margins for acoustics with respect to changes in Mach number, angle of attack, angle of sideslip, and AA/TA elevation. The 3-percent-scale wind-tunnel tests, which were focused on stability and control concerns but included acoustic measurements, predicted an SPL inside the cavity of approximately $147 \mathrm{~dB}$ at Mach 0.85, with energy concentrated over narrow frequency bands. The tests indicate the possibility of two modes: a leading-edge tone at low speeds, and a standing wave tone at high speeds. The 3-percent-scale wind-tunnel tests also indicated that acoustics could be substantially affected by aperture elevation. 


\section{Stability and Control Concerns}

In addition to the cavity resonance concerns, there was a concern that the unsteady flow downstream of the open cavity could reach the empennage, possibly affecting the stability of the airplane or the effectiveness of the elevators or the rudder control surfaces. Some small effects were predicted by the 3-percent-scale wind-tunnel tests. The 7-percent-scale wind-tunnel tests predicted some supersonic flow over the aft fairings and some pressure fluctuations just aft of the aft fairings, but no substantial effects on the empennage.

The 3-percent-scale wind-tunnel tests predicted a small reduction in longitudinal static stability $\left(C_{m_{\alpha}}\right)$ and weathercock stability $\left(C_{n_{\beta}}\right.$ ) during open-door flight of as much as 5 percent and 6 percent, respectively. A small reduction in elevator effectiveness $\left(C_{m_{\check{\delta}}}\right.$ ) was seen when the cavity and telescope were at 60 deg elevation. In addition, the total drag of the airplane was predicted to increase by 2-4 percent, depending on the configuration. While none of the stability and control predictions from the 3-percent-scale wind-tunnel test were of significant concern, there were concerns that the unsteady effects of the cavity may not have been captured accurately downstream, raising the possibility that the effects on the airplane could be more significant than predicted.

\section{Air Data Systems}

Another area of concern during open-door flight testing was the possibility of effects on the air data systems of the SOFIA airplane. Based on experience with downstream flow disturbances affecting air data systems, this possibility needed to be investigated. While the cavity of the SOFIA airplane is significantly aft of the air data system, the extent of the disturbance caused by the open cavity was an unknown.

\section{Investigation Methods}

To evaluate the airplane and provide clearance for an operational lifetime of 20 years, methods were developed to assess concerns and provide envelope clearance for the areas of acoustics, stability and control, and air data. Various types of instrumentation were installed and analysis methods were modified, as appropriate. The acoustics methods and instrumentation used were unique to this application, and as such are described in greater detail below.

\section{A. Acoustics}

In order to detect acoustic resonance or any other pertinent acoustic phenomena, pressure sensors were installed in and around the SOFIA cavity. The locations of these sensors were determined by predicted cavity acoustics, including results from wind-tunnel testing and CFD analysis.

\section{Measurements}

The interior cavity (Fig. 8) had a total of 97 sensors placed along the interior walls where the SOFIA telescope was housed. Initially, 90 pressure sensors were installed. Microphones were installed during the latter phases of flight-testing at locations that provided reasons for interest based on earlier flight data analysis. By the end of the open-door cavity envelope expansion, the sensor suite consisted of 90 Kulite $^{\circledR}$ (Leonia, New Jersey) LLE-2DC-750 pressure transducers and seven Kulite ${ }^{\circledR}$ MIC-1A-LQ-080 microphones. The area of highest SPL activity on the cavity walls occurred on the aft bulkhead of the cavity. Figure 9 is a diagram of the sensor locations relative to the aft bulkhead, which experienced most of the acoustic activity on the cavity walls. The microphones installed on the aft bulkhead were placed to capture areas of notable SPL activity (PA8583m and PA8399m) and the location known to have minimum pressure RMS values, to establish a base noise level (PA8410m). 


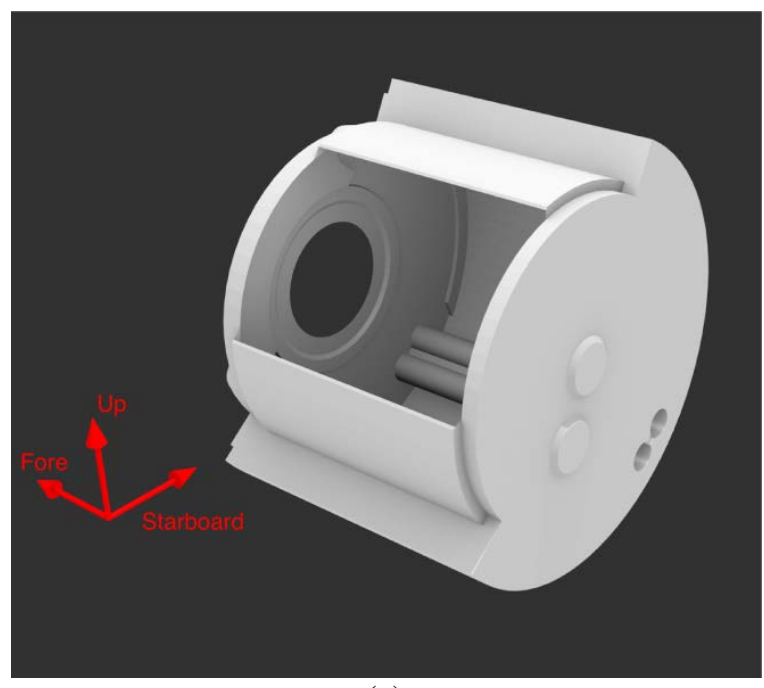

(a)

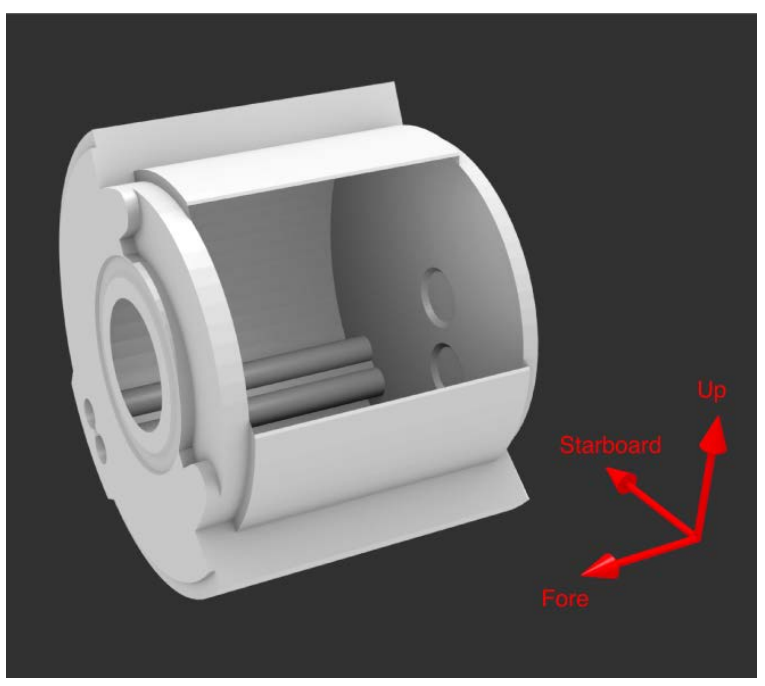

(b)

Figure 8. Isometric view of the SOFIA cavity.

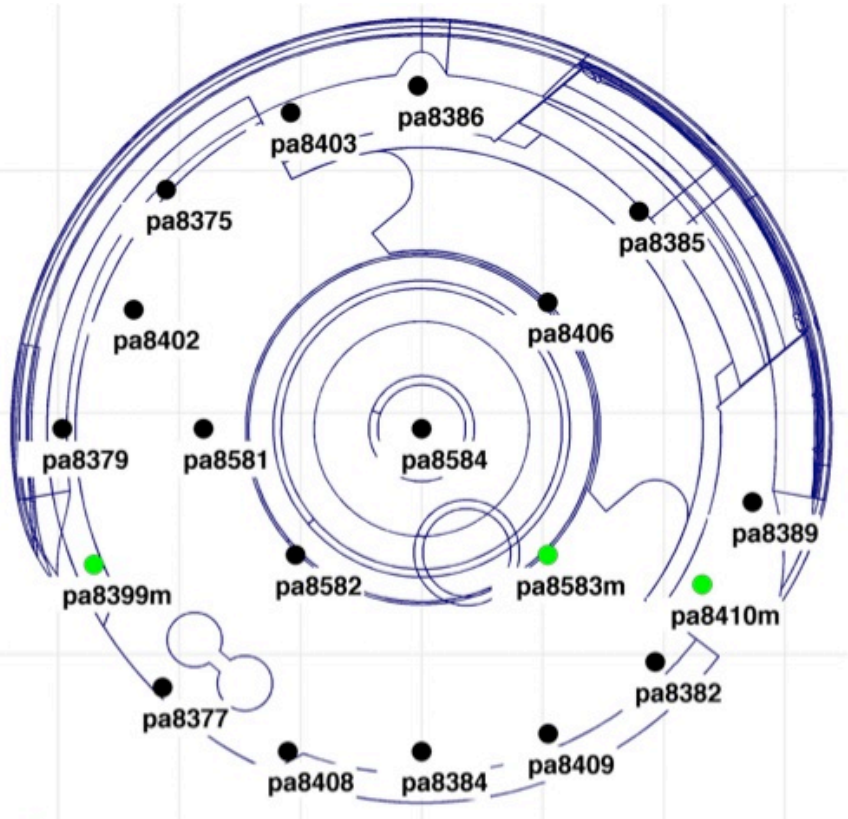

•Green denotes microphones

Figure 9. Cavity aft bulkhead sensor locations.

Five Kulite ${ }^{\circledR}$ LLE-2DC-750 pressure transducers were mounted on the TA spider arms for acoustic monitoring during the Segment 2 envelope expansion flights. A perspective view, illustrating the structure of the TA, can be seen in Fig. 10; the sensor locations are highlighted in red in Fig. 11. The pressure data from these sensors, sampled at 1000 samples per second, were used to characterize the level of interaction between the cavity shear layer and the spider arms. 


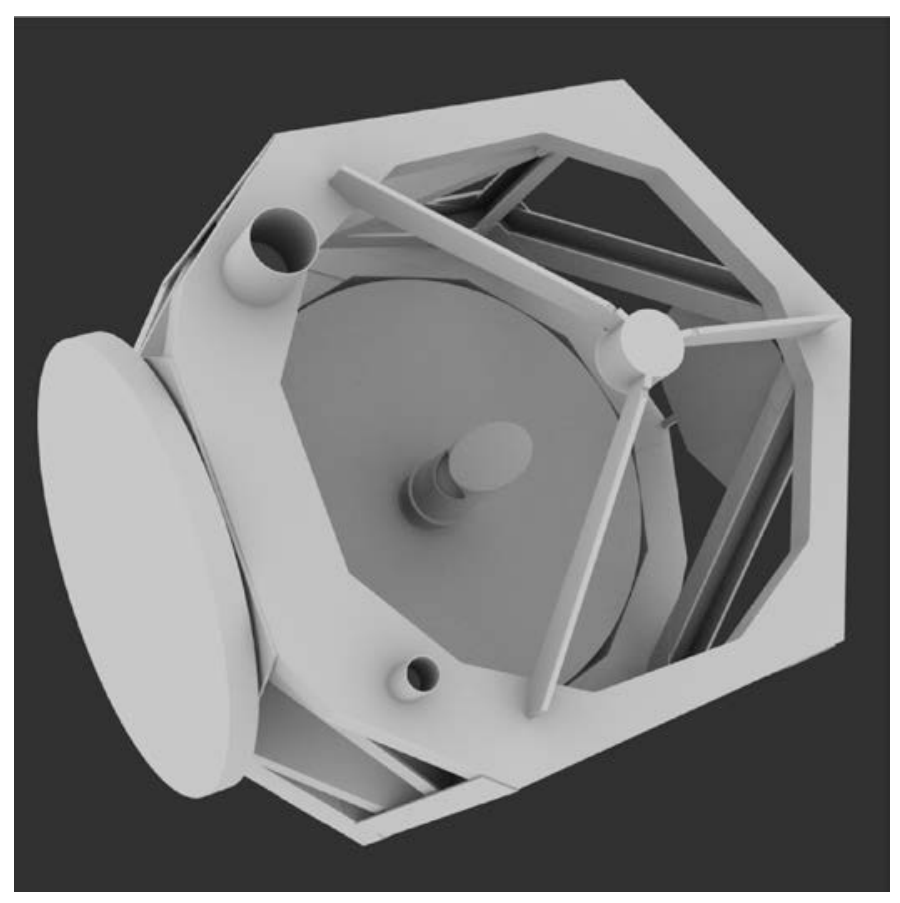

Figure 10. Perspective view of the telescope assembly.

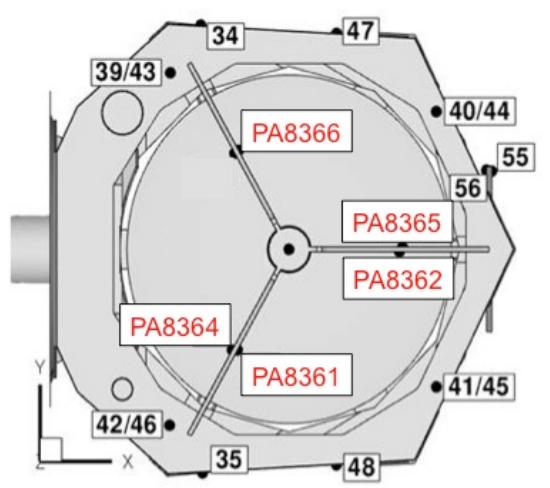

Figure 11. Telescope assembly spider arms acoustic sensor locations.

The internal URD, that is, the side of the URD facing the cavity, had three Kulite ${ }^{\circledR}$ XTL-190A-15A pressure transducers, sampled at 1000 samples per second, for acoustic characterization. The sensors were located at the top, middle, and bottom of the centerline of the URD, as shown in Fig. 12. With the URD fully open, only the bottom sensor (PA8029) was exposed to the cavity. The other two sensors were exposed to airflow in the gap between the inner surface of the URD and the starboard side of the fuselage. 


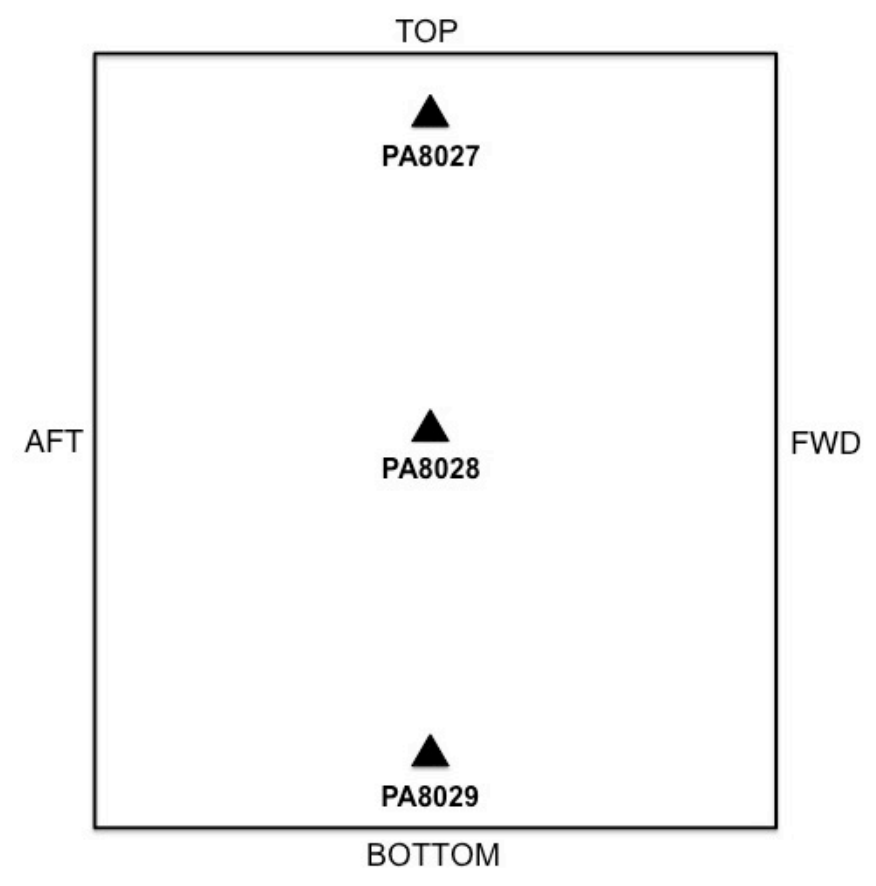

Figure 12. Internal upper rigid door sensor locations.

Acoustic characterization of the internal LFD surface, which is the surface exposed to the cavity, was accomplished using five Kulite ${ }^{\circledR}$ XTL-191B-190-15A pressure transducers that were each sampled at 2000 samples per second. As shown in Fig. 13, three of the sensors were located at the top, middle, and bottom of the centerline of the LFD. The other two sensors were located adjacent to the top center LFD sensor.

TOP

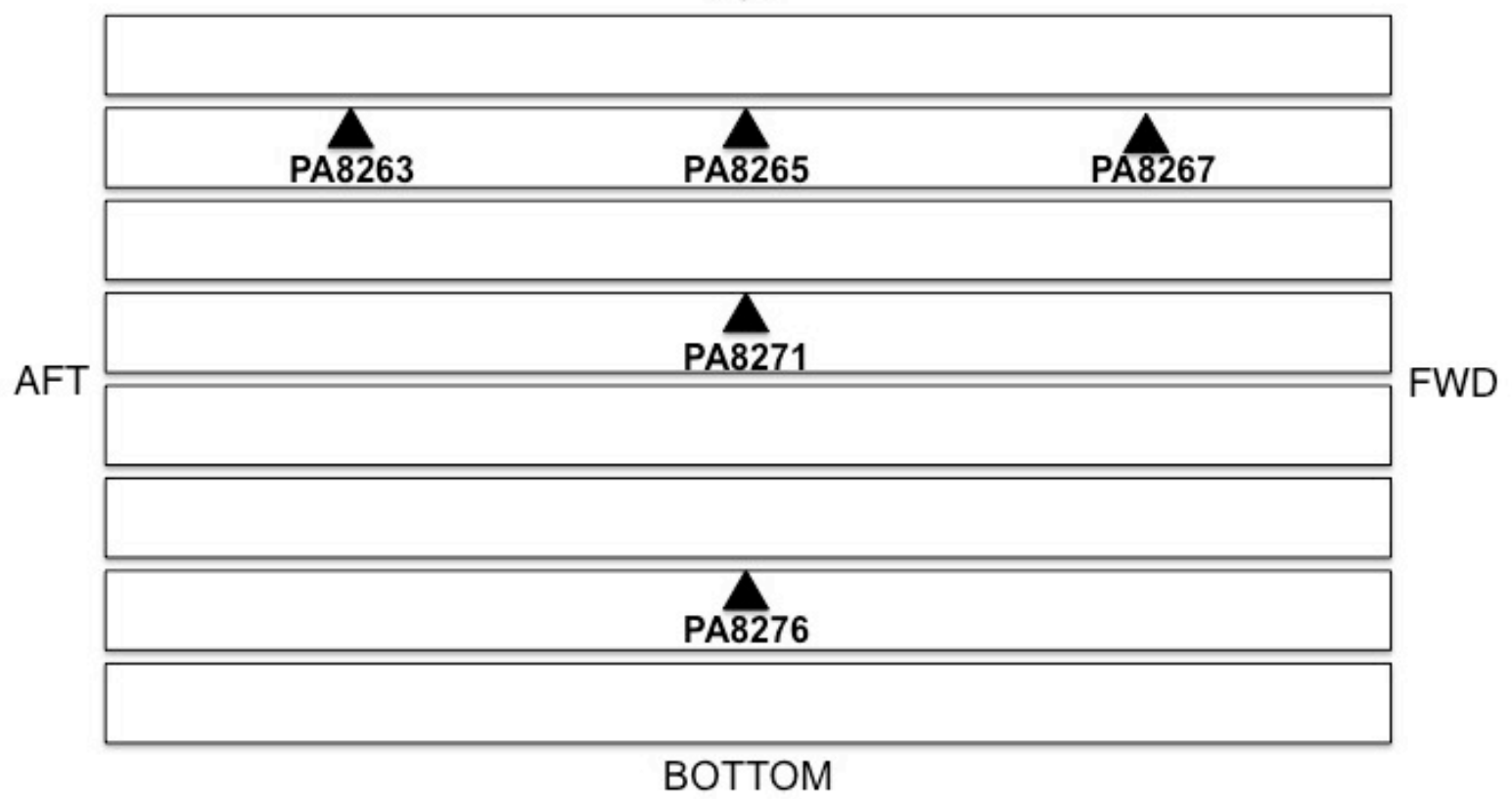

Figure 13. Internal lower flexible door sensor locations. 
Sixteen Kulite ${ }^{\circledR}$ LL-7A-125-15A pressure transducers were used to characterize the acoustic levels on the external AA. This sensor layout is shown in Fig. 14. Eight of the sensors (PA8279, PA8283, PA8285, PA8289, PA8290, PA8296, PA8299, PA8300) were sampled at 5000 samples per second, while the remaining eight sensors were sampled at 1000 samples per second.

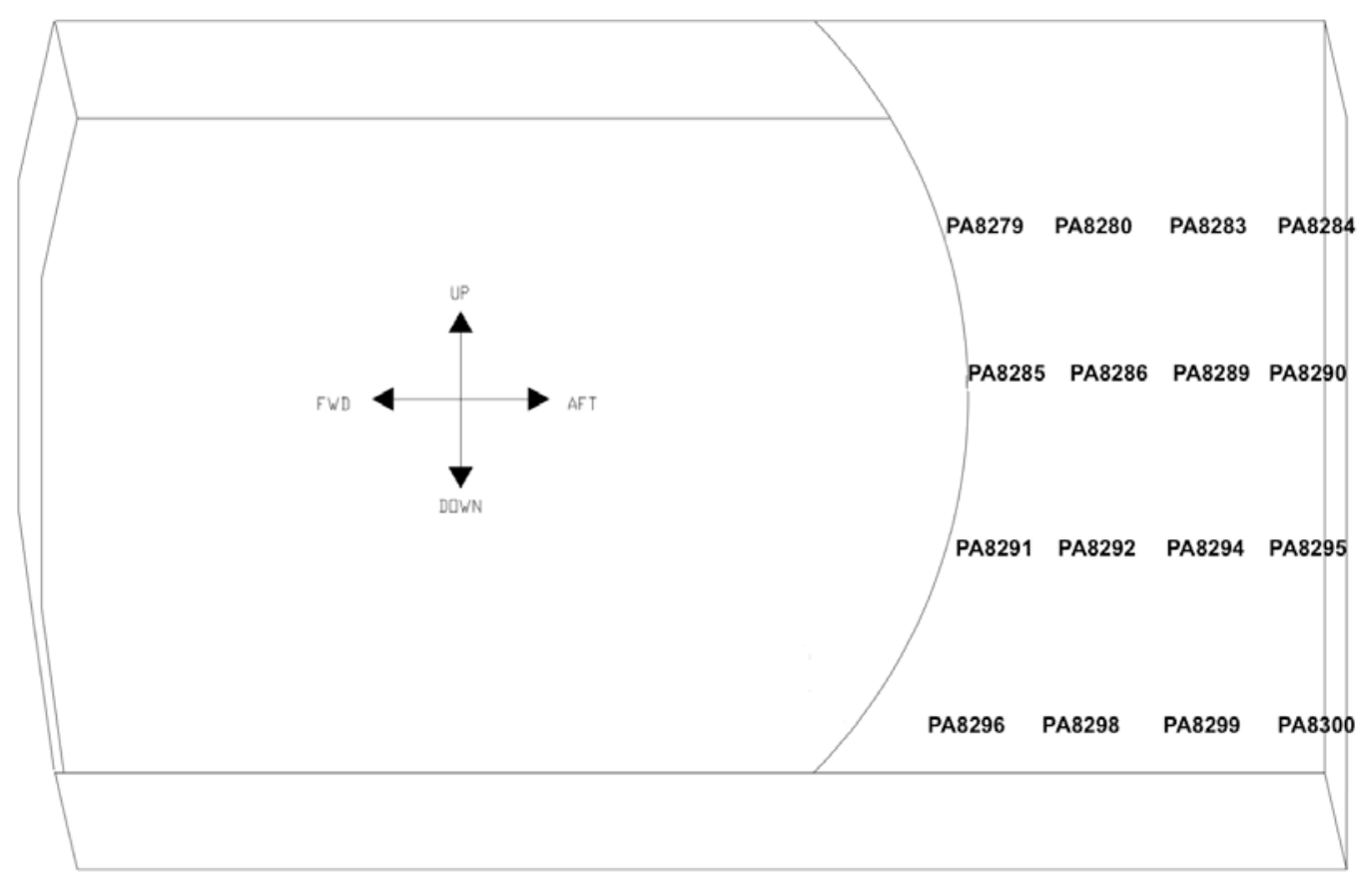

Figure 14. External aperture assembly sensor locations.

The analog-to-digital converter for the entire pressure transducer suite was a 12-bit system with a pressure range from -1.7 to +15.5 psi. The respective data acquisition system channel of each pressure transducer was digitally filtered using a six-pole anti-aliasing filter at a corner frequency of one-fifth the sample rate of its pressure transducer. Such filtering resulted in the sensors sampled at 1000 samples per second having a bandwidth of 0-200 Hz, 2000 samples per second sensors having a bandwidth of 0-400 Hz, and 5000 samples per second sensors having a bandwidth of 0-1000 Hz.

Although the signal conditioning techniques for each pressure transducer were the same, the different frequency ranges, system characteristics, and locations of the sensors resulted in slightly different signal-to-noise ratios. It was, therefore, necessary to note the specific noise floor for each individual sensor location and sampling rate. Table 1 shows the respective SPL base noise levels for each pressure transducer. 
Table 1. SOFIA pressure transducer base noise levels.

\begin{tabular}{|l|l|c|}
\hline \multirow{2}{*}{ Sample rate (samples per second) } & \multicolumn{1}{|c|}{ Surface } & Base noise level (dB) \\
\hline \hline \multirow{2}{*}{1000} & Cavity & 120 \\
\cline { 2 - 3 } & TA & 119 \\
\cline { 2 - 3 } & AA - external & 116 \\
\cline { 2 - 3 } & AA- internal & 113 \\
\cline { 2 - 3 } & URD - internal & 113 \\
\hline \multirow{2}{*}{2000} & LFD - external & 113 \\
\cline { 2 - 3 } & LFD - internal & 112 \\
\cline { 2 - 3 } & URD - external & 129 \\
\hline \multirow{2}{*}{5000} & AA - external & 115 \\
\hline
\end{tabular}

\section{Analysis}

Much consideration was given to measuring the pressure spectrum within and around the cavity. The primary two metrics for acoustic analysis were SPL and PSD. It is important to realize that high pressure levels are not intrinsically dangerous to the SOFIA airplane, whether at discrete frequencies in the spectrum or integrated in the form of a sound pressure level. Rather, it is the influence of the pressure distribution as a dynamic excitation of all structural components, including the TA, AA, URD, and LFD that is important. Since pressure field and structural vibrations are coupled, pressure levels are readily monitored, providing a picture of any resonance within the cavity. The overall objective of the acoustic measurement analyses was to ensure that opening the cavity door, and thereby exposing the interior cavity to the exterior flow field, would not lead to dynamic phenomena that would affect the airworthiness and long-term viability of the SOFIA airplane and the observatory as a whole. For most analyses the primary frequency domain of concern, described in the above section, was expected to cut off at $100 \mathrm{~Hz}$. It was desirable that the pressure instrumentation measure frequency content up to $200 \mathrm{~Hz}$ to ensure the capture of any frequencies of interest, including frequencies with the potential to affect structures or TA components. Sampling was therefore done at no less than 500 samples per second, which corresponds to a Nyquist frequency of $250 \mathrm{~Hz}$. All sensors used for acoustics analysis were sampled at 1000 samples per second or greater.

One-second average SPL was calculated for each pressure sensor, as shown in Eq. 1:

$$
S P L=20 \log _{10}\left(\frac{p_{R M S}}{p_{\text {ref }}}\right)
$$

where $p_{\text {ref }}=2.9 \mathrm{e}-9 \mathrm{psi}$, and $p_{R M S}$ is the RMS unsteady pressure in psi units, as shown in Eqs. (2) and (3):

$$
\begin{gathered}
p_{R M S}=\sqrt{\frac{1}{n} \sum_{i=1}^{n}\left(p_{i}-p_{\text {avg }}\right)^{2}} \\
p_{\text {avg }}=\frac{1}{n} \sum_{i=1}^{n} p_{i}
\end{gathered}
$$

The quantity $p_{a v g}$ is the average pressure over the sample window, and the value $n$ is the number of measurements in the window. The $p_{R M S}$ calculation uses a sample window equivalent to one second of pressure values, ending with the sample of interest. The window shifts by one sample, continuously adding the newest data point and discarding the oldest.

The frequency content of the acoustic measurements was observed through PSD analysis. PSD was estimated using Welch's method. ${ }^{7}$ Welch's method splits the data into overlapping segments, computes modified periodograms of the overlapping segments via a fast Fourier transform (FFT), and averages the resulting 
periodograms to produce the PSD estimate. Similar to SPL calculations, the sample window size was equivalent to one second of samples. The full data set was divided into an integer number of segments whose size was equal to the sample window size with overlap equal to 32 samples. Each segment was windowed with a Hanning window. The number of points used in the FFT was the next power of two greater than the length of the segment size. For PSD analysis, the oscillatory pressure values, $p_{A C}$, relative to the sample window average were used instead of absolute measurements for the $i$ th measurement in the window, as shown in Eq. (4).

$$
\left(p_{A C}\right)_{i}=p_{i}-p_{\text {avg }}
$$

\section{B. Stability and Control}

Standard parameter estimation techniques ${ }^{8,9}$ were used to determine the stability and control derivatives of the SOFIA airplane. The primary tool used for parameter estimation was a program called $p E s t .{ }^{10}$ Cramér-Rao bounds, approximations of the estimated parameter variances, were used to provide a relative assessment of the accuracy of the results. ${ }^{8}$ The goal of the parameter estimation work was to determine if the modifications to the aircraft negatively impacted its stability and the effectiveness of its control surfaces. Of particular interest were the effects on the elevator and rudder surfaces and the changes in directional stability.

Flight data from the baseline flight phase were obtained, so that parameter estimation work could be performed for the unmodified aircraft. The analysis was performed between Segments 1 and 2 of the SOFIA flight tests. The maneuvers used during the baseline flight phase consisted of three pulses in alternating directions, essentially doublets with an extra pulse tacked on. There were some variations in pulse lengths. Two maneuvers were flown at each flight condition during the baseline flights.

Doublets were the only maneuvers used for parameter estimation during the SOFIA flight-test phases. Longitudinal axis derivatives were estimated using pitch doublets. Lateral-directional derivatives were estimated using maneuvers that consisted of a yaw doublet immediately followed by a roll doublet. Maneuvers were typically performed in sets of three to provide repeatability. During Segment 2, the starting directions of the yaw-roll doublets were alternated for each repeat, as a way to potentially indicate asymmetries in the aerodynamics.

\section{Flight-Test Results}

Throughout Segments 0, 1, and 2, 35 flights were completed to expand the flight envelope for the SOFIA airplane and collect data to clear the airplane for operational service. Substantial amounts of data were collected in the areas of acoustics, stability and control, and air data. None of the air data systems were found to have been affected by the SOFIA modifications. The research FADS system was successfully calibrated. Both the production pitot-static system and the research FADS system functioned nominally throughout the various flight segments.

\section{A. Acoustics}

Acoustics data were collected throughout the flight envelope of the SOFIA airplane, over the full range of AA/TA elevations at various URD positions during Segment 2 flights. These data were used to characterize the cavity acoustic environment throughout the flight envelope. Acoustics are discussed with respect to factors affecting overall SPL, the characteristics of PSDs at various locations, and other areas of interest during the flights. The areas that are discussed are the TA spider arms, the URD, the LFD, the AA, and the cavity walls.

\section{Flight Condition Effects on Sound Pressure Level}

A series of test points were flown throughout envelope expansion during Segment 2 to characterize the acoustic field within and around the cavity at scientific observation flight conditions and off-design conditions. For the purposes of this paper, an altitude of 42,000 ft and Mach 0.85 will be considered the nominal scientific observation flight condition. Any flight conditions or cavity configurations that resulted in acoustic resonance or any other risk to the structures in and around the cavity needed to be identified. During normal aircraft operations, that is, steady climb, cruise, and descent, there were no acoustic anomalies experienced within the cavity. As expected, pressure levels generally increased with dynamic pressure, showing an increase with Mach number at constant altitude and a decrease with altitude at a constant Mach number.

The TA spider arm sensors experienced an average SPL that varied between 120 and $131 \mathrm{~dB}$, with the highest average SPL occurring at a flight condition of an altitude of 15,000 ft and Mach 0.59. The aft spider arm sensors, PA8365 and PA8362 in Fig. 11, consistently registered the highest SPL of all the spider arm sensors. These two sensors had the most interaction with the cavity shear layer due to their location farther aft of the cavity leading edge. 
The average SPL on the internal URD varied between 124-137 dB, while the average SPL of the internal LFD varied between 118-128 dB. Two of the URD sensors displayed interesting behavior at various flight conditions. Sensor PA8027, located at the center top of the URD, registered up to $146 \mathrm{~dB}$ at the highest dynamic pressure test point (Mach 0.69 at 15,000 ft). This sensor was exposed to the flow on the starboard portion of the fuselage behind the forward fairing when the URD was open. The right inboard LFD sensor (PA8267) consistently registered the highest SPL of all of the internal LFD sensors.

The AA ramp is exposed to the flow when the URD is open and serves as the reattachment region for the cavity shear layer. Consequently, as expected, the highest SPL of all of the surfaces were observed on the AA. The average SPL on the AA ramp ranged from 143-155 dB during the Segment 2 envelope expansion. The aft surface of the ramp typically displayed the highest SPL, with individual sensors approaching $161 \mathrm{~dB}$ at the altitude of 15,000 ft and Mach 0.69 flight condition. As stated above, the AA was instrumented with a combination of $200 \mathrm{and} 1000 \mathrm{~Hz}$ bandwidth sensors. The energy content due to the additional bandwidth was determined to be, on average, 0.4-1.4 dB, or roughly $0.13-0.45$ percent of the average SPL using all 16 sensors. Since the energy content due to the additional bandwidth was negligible, no filtering was applied to the $1000-\mathrm{Hz}$ bandwidth sensors when they were averaged with the $200-\mathrm{Hz}$ bandwidth sensors.

Measurements on cavity walls as a whole did not demonstrate an adverse acoustic atmosphere. The average SPL level of the cavity walls was generally just above the base noise level of the sensors shown in Table 1, less than $123 \mathrm{~dB}$; however, there exists a region within the cavity that is referred to as the "hot spot" due to the high SPL levels it experienced in relation to the rest of the cavity. Unsurprisingly, the hot spot was located on the aft bulkhead portion of the cavity walls, closest to the side of the URD. The sensors located in this area were PA8385, PA8389, PA8386, and PA8406, as seen in Fig. 9. With the URD 100-percent open, the highest average SPL across the entire bulkhead was approximately $127 \mathrm{~dB}$. This occurred at flight conditions of a 15,000-ft pressure altitude and Mach 0.69 with the AA at an elevation of $23^{\circ}$. At these same conditions, however, the hot spot experienced an average $133 \mathrm{~dB}$.

At the nominal scientific observation flight condition, with the URD at 100-percent, the highest SPL values occurred when the AA was at $57^{\circ}$. Figure 15 shows the average SPL at the nominal scientific observation flight condition for two different AA settings. The hot spot is visible toward the upper right-hand part of the image. As the AA setting increases, the area of maximum SPL can be seen shifting slightly.

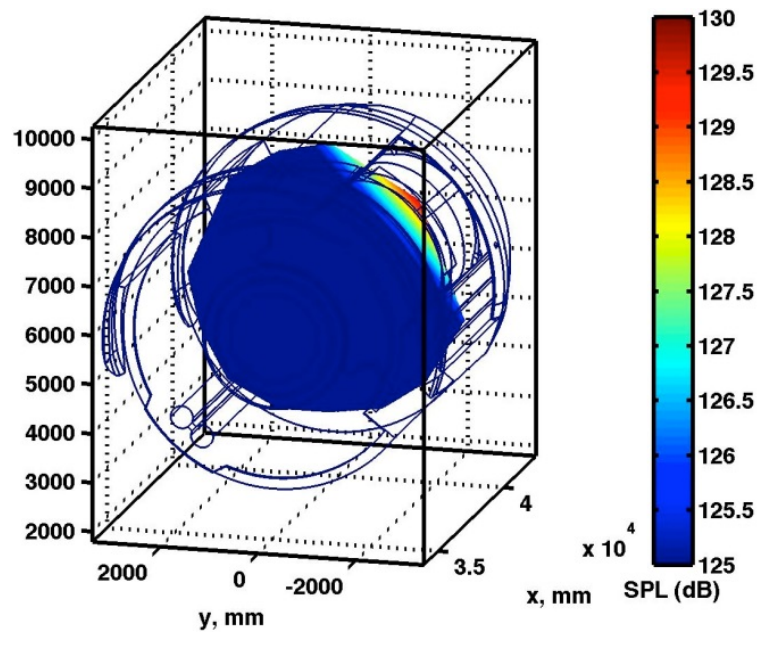

(a)

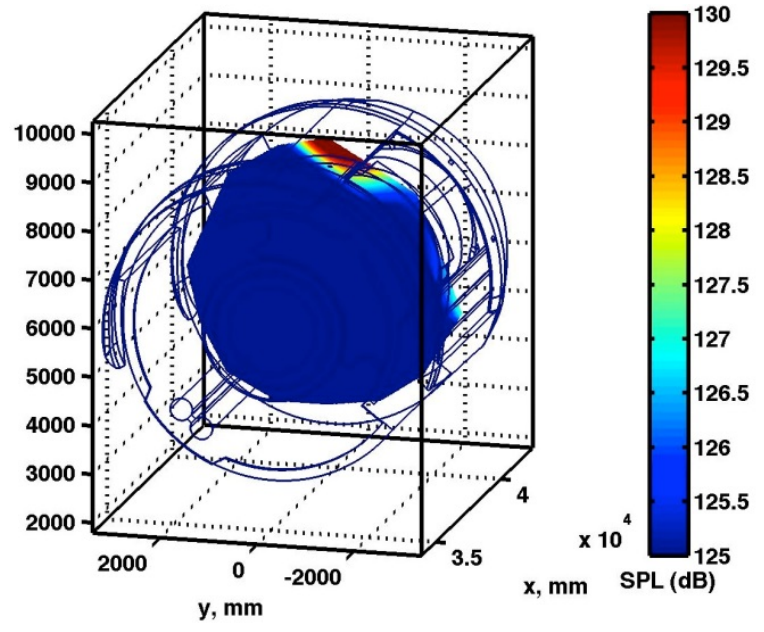

(b)

Figure 15. Cavity aft bulkhead hot spot at the nominal science observation flight condition. (a) Aperture assembly $=30^{\circ}$ and (b) $57^{\circ}$.

Figure 16 shows the change of SPL at the cavity hot spot due to flight conditions for AA settings of $23^{\circ}, 40^{\circ}$ and $57^{\circ}$. A moving average algorithm was used to fit the test point data so that the trends could be easily identified. An AA elevation of $57^{\circ}$ typically resulted in the highest SPL levels at any given flight condition; however, there is no discernable trend regarding smaller AA settings. Pressure levels never reached magnitudes capable of causing damage at any flight condition. 




Figure 16. Sound pressure level versus Mach. Cavity hot spot, upper rigid door at $100 \%$.

As mentioned above, several wind-tunnel tests had been performed to predict the effects of flight condition on the cavity acoustic field. Figure 17 shows a comparison between data from the flights and scaled data from the final 7-percent-scale wind-tunnel test series (SOFIA document 05-N-19980220 - 001, "SOFIA V Design Validation Test Final Report," dated April 15, 1998). While the absolute values from the test do not necessarily match the flight data, the trends are similar.

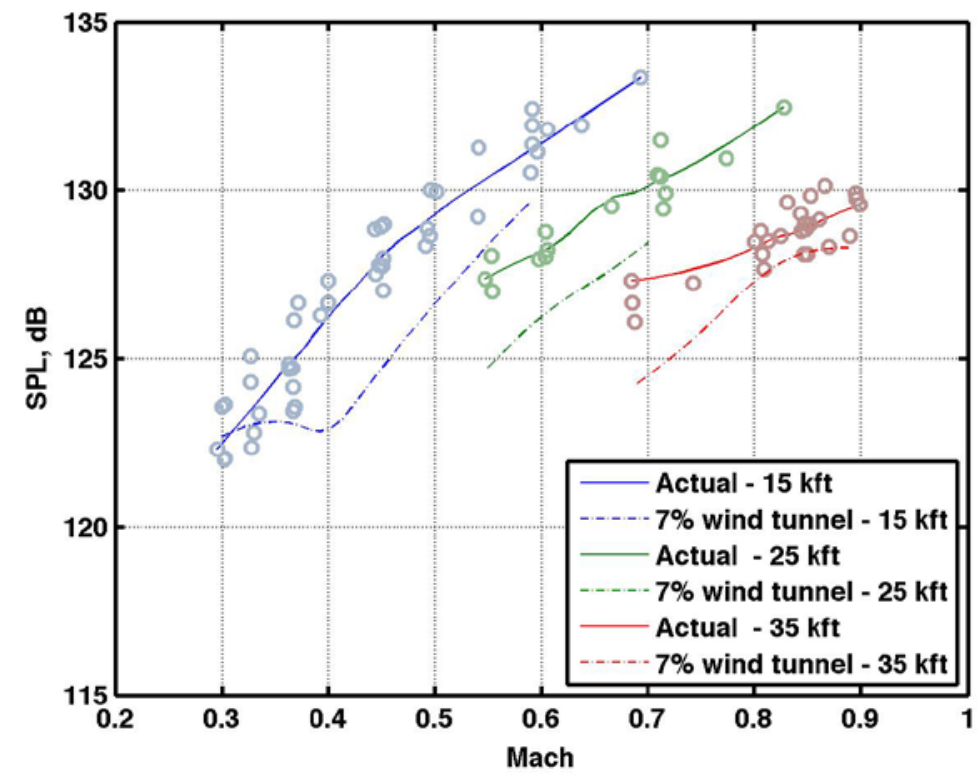

Figure 17. Scaled 7-percent wind tunnel sound pressure level versus Mach. Cavity hot spot. Upper rigid door at 100 -percent and aperture assembly at $23^{\circ}$. 
2. Configuration Effects on Sound Pressure Levels

Different configurations and settings of the various SOFIA components changed the acoustic characteristics inside and around the cavity. These different configurations included the position of the URD, the elevation angle of the AA when aligned with the TA, and also the offset when the TA and AA were misaligned.

For different AA elevations ranging from 23 to $57^{\circ}$ at a given flight condition, the measured TA SPL peaked at an AA elevation of $40^{\circ}$, then decreased as the AA was moved to higher or lower elevations. The peak was less distinguishable with the increase of $\bar{q}$, becoming indiscernible at the nominal scientific observation flight condition. The change in AA elevation had an insignificant effect (approximately one dB) on the measured SPL level of the cavity.

Figure 18 shows the TA SPL variation due to URD position. For AA elevations of 23 and $57^{\circ}$, the overall SPL increased by approximately $3 \mathrm{~dB}$ as the URD was moved from 10-percent open to fully open. At an AA elevation of $40^{\circ}$, the SPL increased by approximately $6 \mathrm{~dB}$.

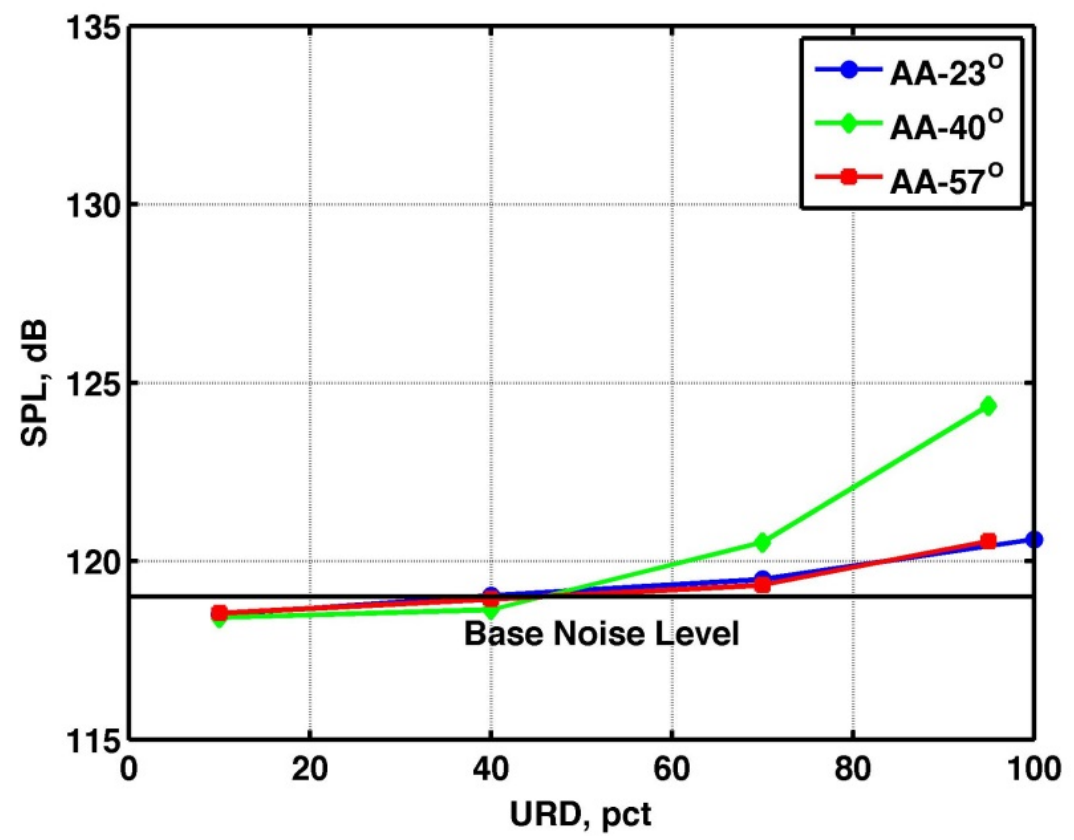

Figure 18. Sound pressure level versus door opening for TA spider arms at the nominal scientific observation flight condition.

Extensive TA misalignment testing was performed at an altitude of 35,000 ft over a Mach number range of 0.83-0.87. Two types of misalignment were studied: TA elevation angle and TA cross elevation. During the TA elevation misalignment points, the AA was parked at a constant elevation while the TA was moved to various elevations below and above the AA setting. As shown in Fig. 19, the overall SPL increased as the TA elevation was moved above the fixed AA elevation angle. The opposite effect occurred when the TA was moved to elevations below the AA elevation. 


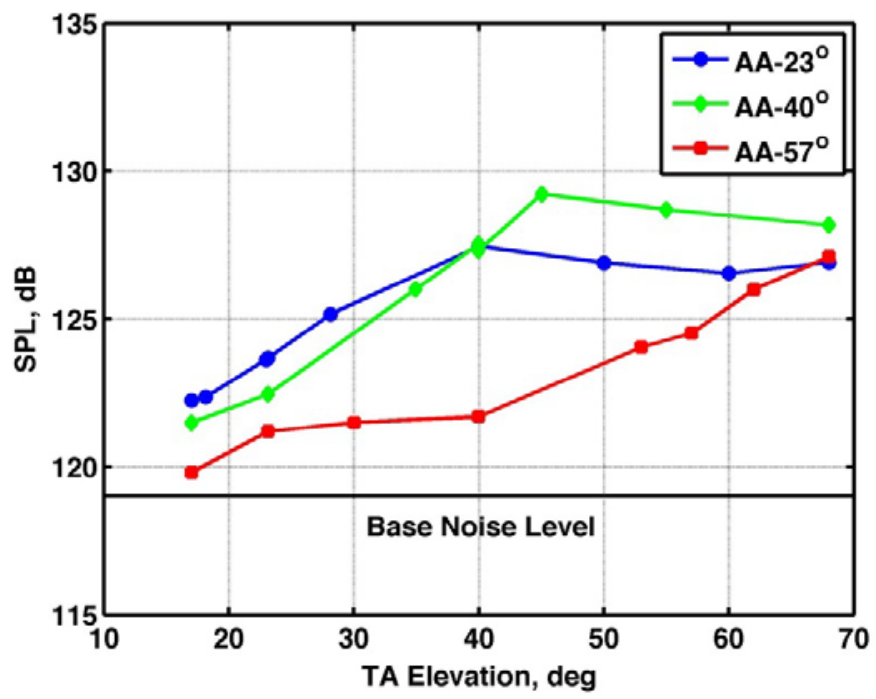

Figure 19. Telescope assembly spider arm sound pressure level variation due to telescope assembly misalignment at the nominal scientific observation flight condition.

The TA cross-alignment testing involved both positive and negative cross-elevation changes. Positive cross elevation displacement moved the TA farther out into the cavity shear layer, whereas negative cross-elevation displacement moved the TA back into the cavity. At positive cross elevations, the overall spider arm SPL increased by approximately $1 \mathrm{~dB}$; at negative cross elevations, the SPL decreased by approximately $1 \mathrm{~dB}$.

The external AA showed sizeable variations in SPL for different door positions. As one might expect, the SPL increased as the URD opened because the external AA became exposed to the outside flow.

Changes in door position for a given flight condition had negligible effect on the overall SPL of the URD and LFD and did not generally result in large changes in SPL of the cavity walls. The SPL consistently increased with URD opening. A change of less than $5 \mathrm{~dB}$ between 10-percent and 100-percent URD opening was typical. The SPL levels due to change in AA at those same conditions resulted in even less variance. Figure 20 shows the variation of SPL with change in door opening and AA elevation at an altitude of 42,000 ft and Mach 0.85, the nominal scientific observation flight condition.

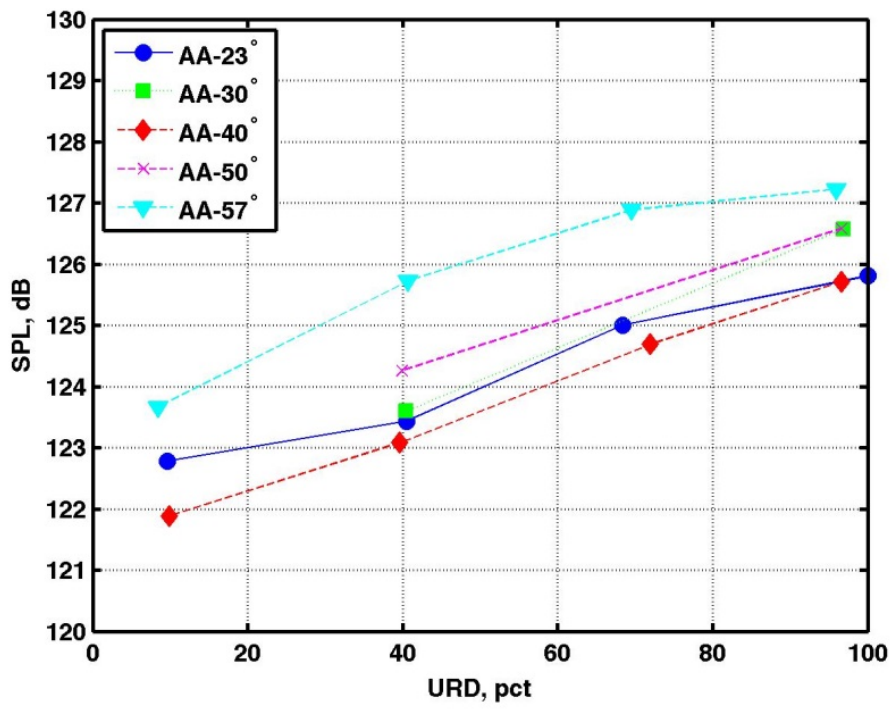

Figure 20. Sound pressure level versus door opening for the cavity hot spot at the nominal scientific observation flight condition.

American Institute of Aeronautics and Astronautics 


\section{3. $\quad$ Sideslip Effects on Sound Pressure Levels}

Variations in angle of sideslip resulted in overall SPL changes for the cavity and other surfaces, with some surfaces being affected more than the others. During sideslip maneuvers, positive sideslip placed the cavity on the lee side of the flow whereas negative sideslip placed the cavity on the upstream side of the flow. The highest SPL levels seen during sideslip maneuvers generally occurred at low-altitude, low-speed test points due to the ability to reach large sideslip angles. Sideslip angle effects were less prevalent at higher speeds and altitudes due to the smaller available sideslip.

Figure 21 shows the effect of sideslip angle on the overall spider arm SPL at the nominal scientific observation flight condition. The spider arm sensors for the $40^{\circ}$ and $57^{\circ}$ AA elevations displayed peaks of approximately $4 \mathrm{~dB}$ above baseline levels as the airplane sideslip angle changed from positive to negative. This peak was not present with the AA at its lowest elevation of $23^{\circ}$.

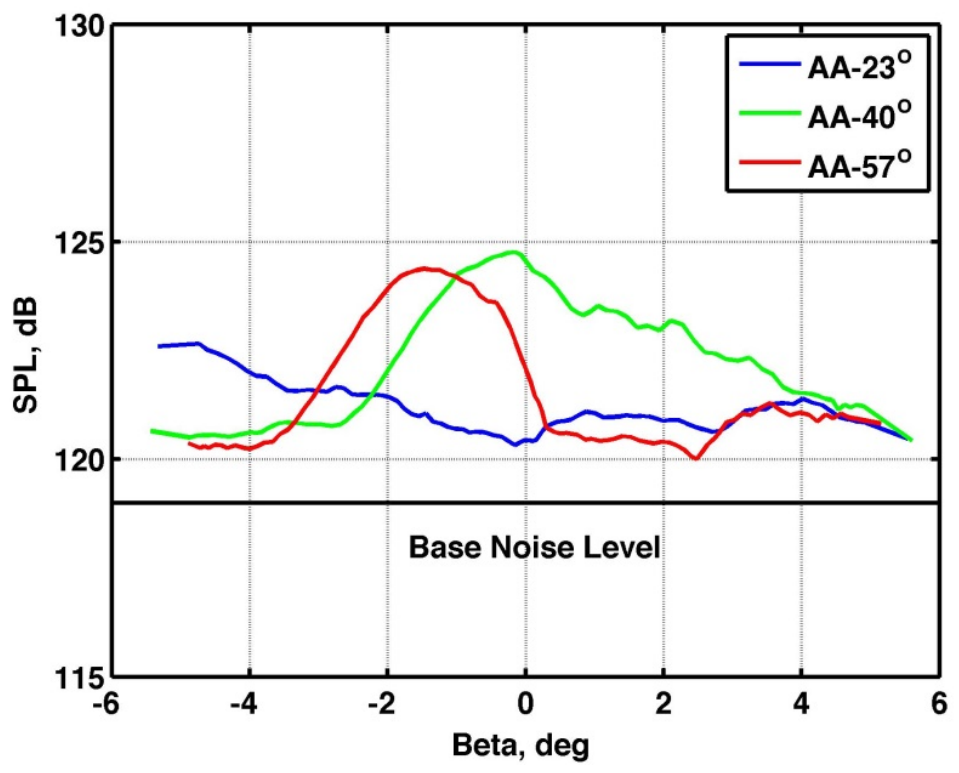

Figure 21. Telescope assembly spider arm sound pressure level variation with sideslip angle at the nominal scientific observation flight condition.

Aircraft sideslip had only minor effects on the overall SPL of the internal URD and internal LFD; not enough to be considered of any significance.

At the nominal scientific observation flight condition, the SPL variation on the AA due to sideslip was on the order of $2 \mathrm{~dB}$, as shown in Fig. 22. At this flight condition, both the $23^{\circ}$ and $40^{\circ}$ AA elevations showed an increase in SPL with sideslip, whereas the $57^{\circ}$ elevation showed a decrease in SPL with positive sideslip, and an increase in SPL with negative sideslip. 


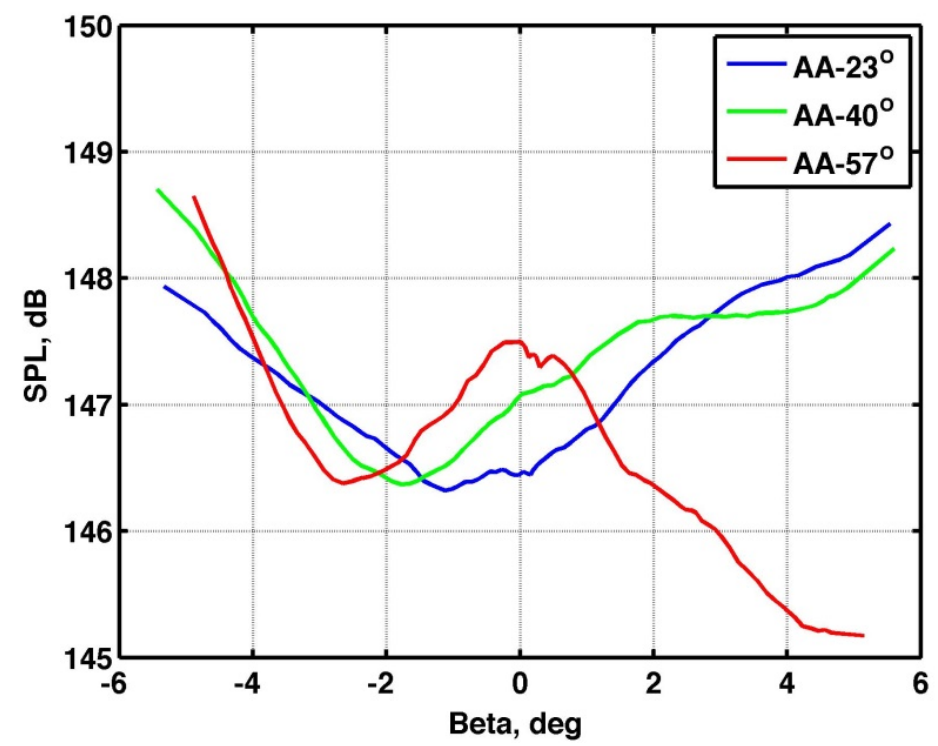

Figure 22. External aperture assembly sound pressure level variations with sideslip angle at the nominal scientific observation flight condition.

Figure 23 shows the effect of sideslip angle on the cavity hot spot at relatively low dynamic pressure flight conditions. For these flight conditions, off-baseline (non-zero) sideslip angles in both the positive and negative directions generally resulted in higher SPL, with the highest levels observed at maximum negative sideslip for AA settings above $30^{\circ}$, and highest levels observed at maximum positive sideslip for AA settings $30^{\circ}$ and below. These test conditions provided the highest in-flight SPL in the cavity, with one sensor within the hot spot (PA8385) reaching just over $145 \mathrm{~dB}$. There was no aircraft structural response to the 145 -dB event. Figure 24 shows the pressure PSD for the event. As can be seen in the figure, there were no substantial frequency peaks during this event. At the nominal scientific observation flight condition, the angle of sideslip has insignificant effects on the cavity hot spot SPL. For this flight condition, the angle of sideslip does affect SPL level, but the effects are not consistent for different AA/TA elevations.

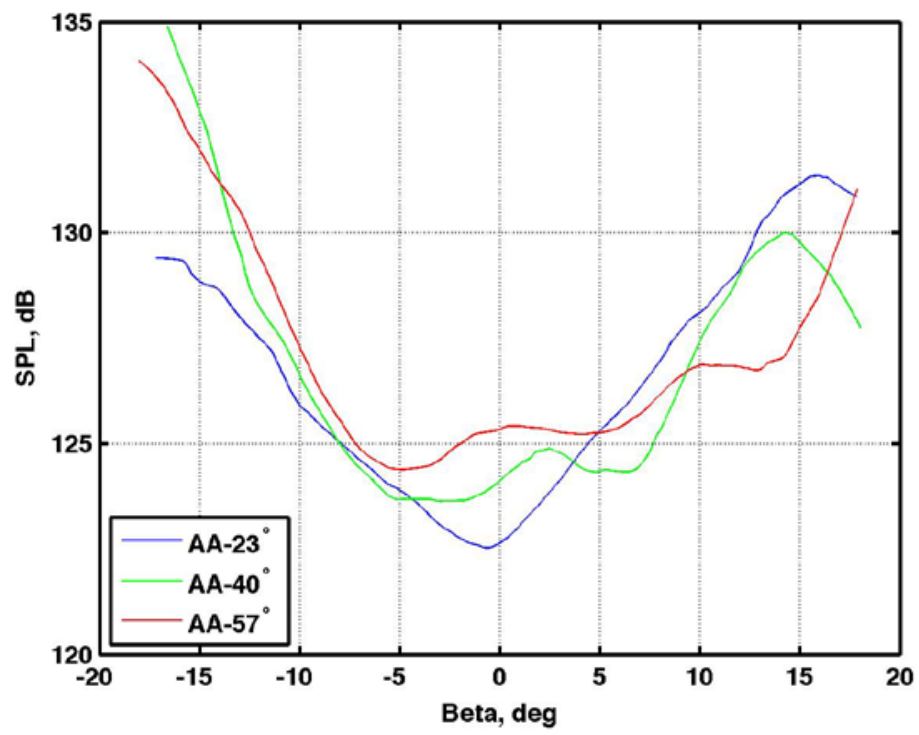

Figure 23. Sound pressure level versus angle of sideslip for the cavity hot spot at an altitude of $15,000 \mathrm{ft}$ and Mach 0.33. 


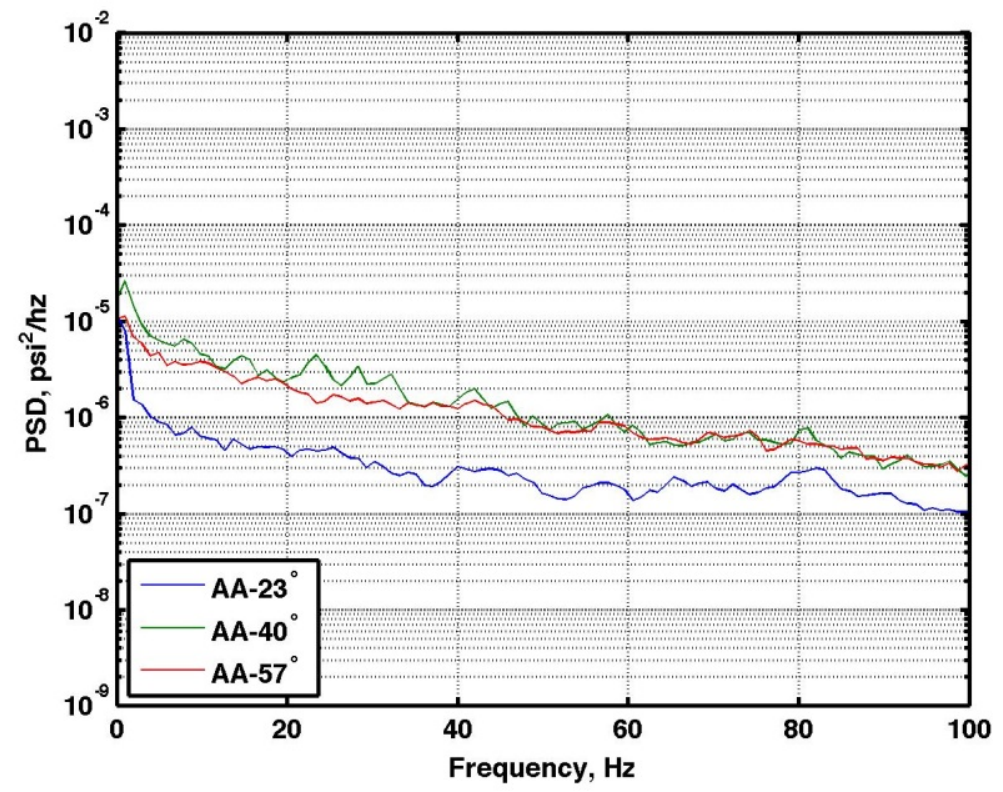

Figure 24. Power spectral density analysis for negative sideslip for the cavity hot spot at an altitude of 15,000 ft and Mach 0.33.

\section{Power Spectral Density Analysis}

The SOFIA airplane modifications associated with the cavity (including the fairings, CDS, and the cavity itself) were designed to reduce the tendency for shear layer ingestion to generate a resonance known as a Rossiter mode. ${ }^{13}$ When a Rossiter mode occurs, certain frequencies within the acoustical spectrum exhibit significantly higher signal levels. The actual frequencies at which these resonances occur depend on the geometry of the opening and the speed of the aircraft. Thus, it is quite conceivable that in some operational phases these frequencies will be very close to the natural frequencies of the aircraft structure or the telescope assembly. Knowledge of how the PSD of the acoustic environment within and around the cavity varies with airspeed was therefore critical to identifying whether flying SOFIA with the door open would result in acoustic resonance or any other risk to the airplane structure.

The frequency content of the TA spider arm and external AA sensors was observed to be broadband in nature throughout open-door envelope expansion. On the internal URD, sensor PA8027 displayed a broad peak around $29 \mathrm{~Hz}$ at an AA elevation of $23^{\circ}$, as shown in Fig. 25. At a $40^{\circ}$ AA elevation, the peak begins to flatten out, and at $57^{\circ}$ the peak is no longer present. The $57^{\circ}$ AA elevation produced some low amplitude frequency peaks at 145 and $180 \mathrm{~Hz}$ on the middle and bottom centrally located LFD sensors (PA8271, PA8276). Figure 26 shows the two frequency peaks in the PSD of sensor PA8276. 


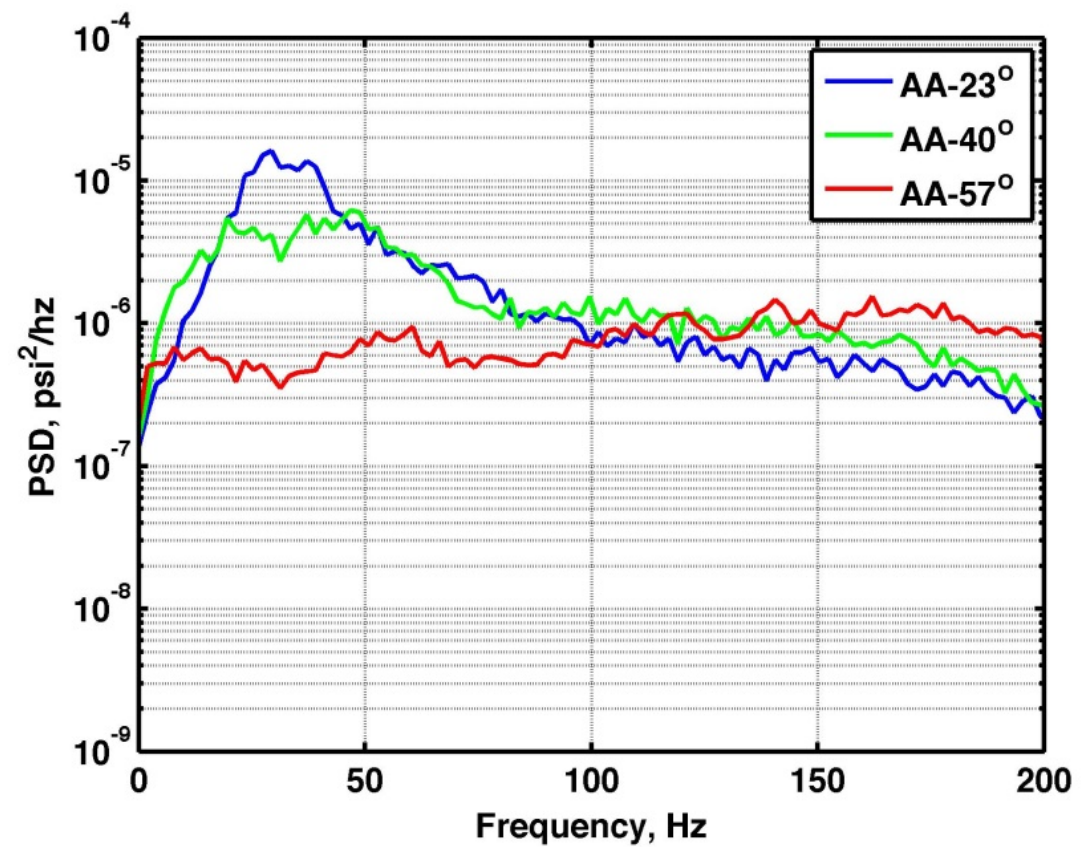

Figure 25. Power spectral density for the internal upper rigid door at sensor PA8027 at the nominal scientific observation flight condition.

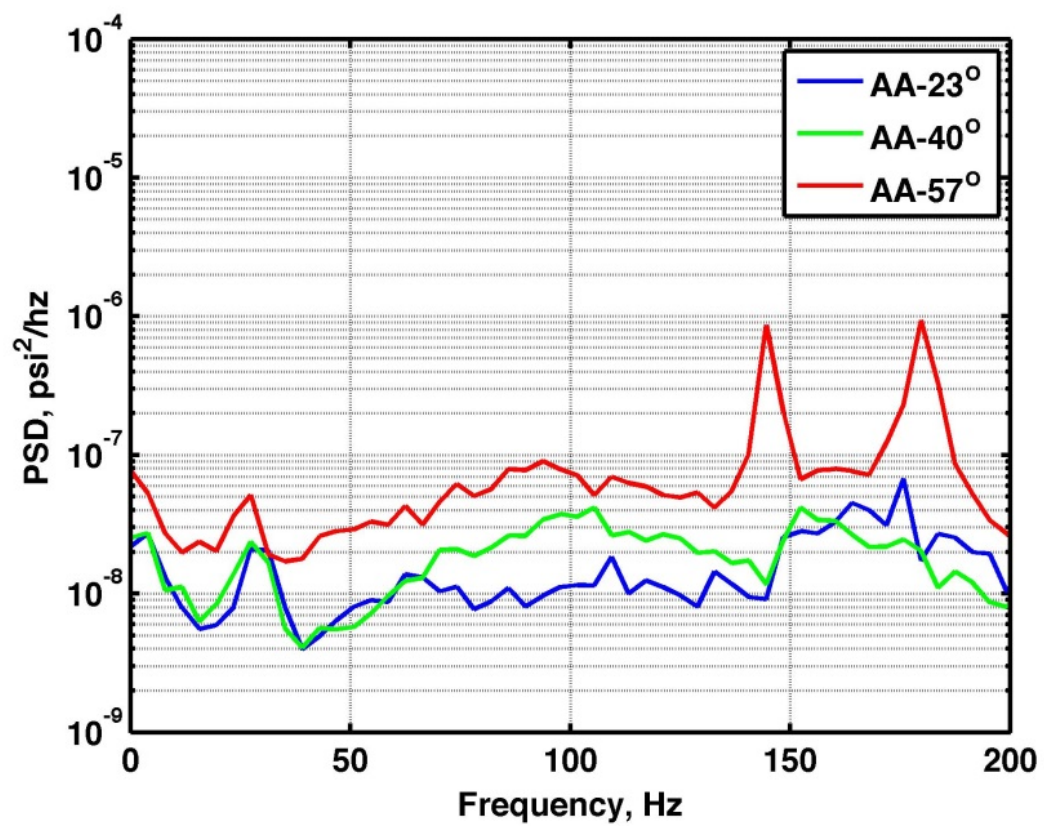

Figure 26. Power spectral density for lower flexible door sensor PA8276 at the nominal scientific observation flight condition.

Within the cavity, no tones that excited a structural response were discovered during open-door envelope expansion and flight-testing. The frequency content observed within the cavity was generally broadband in nature for the majority of the flight conditions and cavity configurations. The $23^{\circ}$ AA elevation, however, occasionally produced a low amplitude frequency peak at $42 \mathrm{~Hz}$, pronouncedly located at the cavity hot spot. The peak was most 
prominent at a URD opening of 40-percent throughout the test envelope, and was even noticeable at a URD opening of 10-percent, at 42,000 ft and Mach 0.85 .

\section{5. $\quad$ Open-Door Landings}

Prior to the planned open-door landing test points, the CDS failed in an open position on several occasions. Five open-door landings were executed due to URD malfunctions; four occurred with an AA elevation of $23^{\circ}$. Of those four, two landings took place with the URD at 40 percent, while the other two were at 70 percent and 100 percent. Another open-door landing occurred with an AA elevation of $40^{\circ}$ and the URD at nearly 100 percent. During each of the landings the cavity experienced a flow-induced resonance. Rossiter mode resonance occurred, beginning as the airplane slowed to 140 knots calibrated airspeed (KCAS). The amplitudes were lower at the approach speed and had frequencies that were approximately $15-20 \mathrm{~Hz}$, corresponding to the second Rossiter mode. As the airplane landed and rolled out, the speed was reduced further to approximately 70 KCAS and the frequencies dropped to approximately 7-8 Hz. This is the first Rossiter mode. Between 140 and 70 KCAS strong resonance and a transition in Rossiter modes were observed, with the SPL reaching a maximum value of $143 \mathrm{~dB}^{2}$ The PSD analysis is shown in Fig. 27.

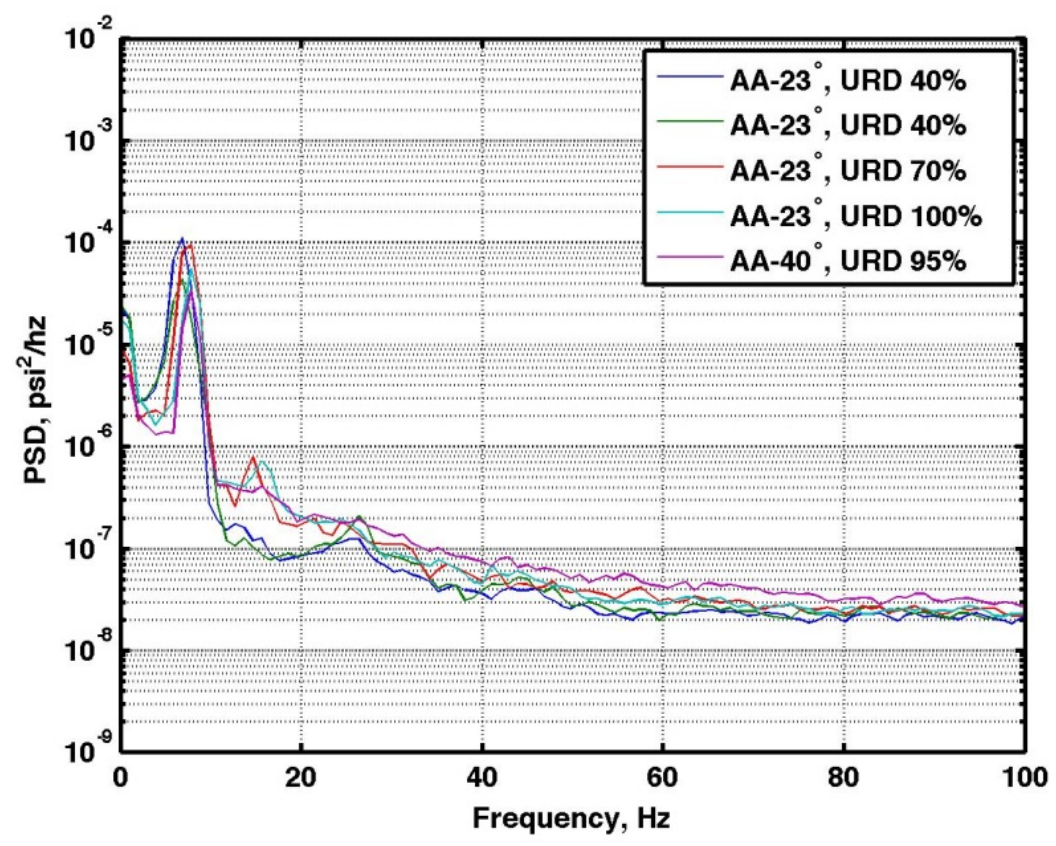

Figure 27. Power spectral density analysis of open-door landings for the cavity hot spot.

A maximum SPL of $143 \mathrm{~dB}$ was measured on the aft bulkhead. Figure 28 shows the variation in SPL as the airplane landed and decelerated (shown from right to left). Also shown is the theoretical maximum SPL that could be generated based on the acoustic energy available from dynamic pressure. The theoretical maximum SPL was calculated from Eq. (5).

$$
S P L_{\max }=20 \log _{10}\left(\frac{1}{2 \sqrt{2}} \frac{\bar{q}}{p_{\text {ref }}}\right)
$$




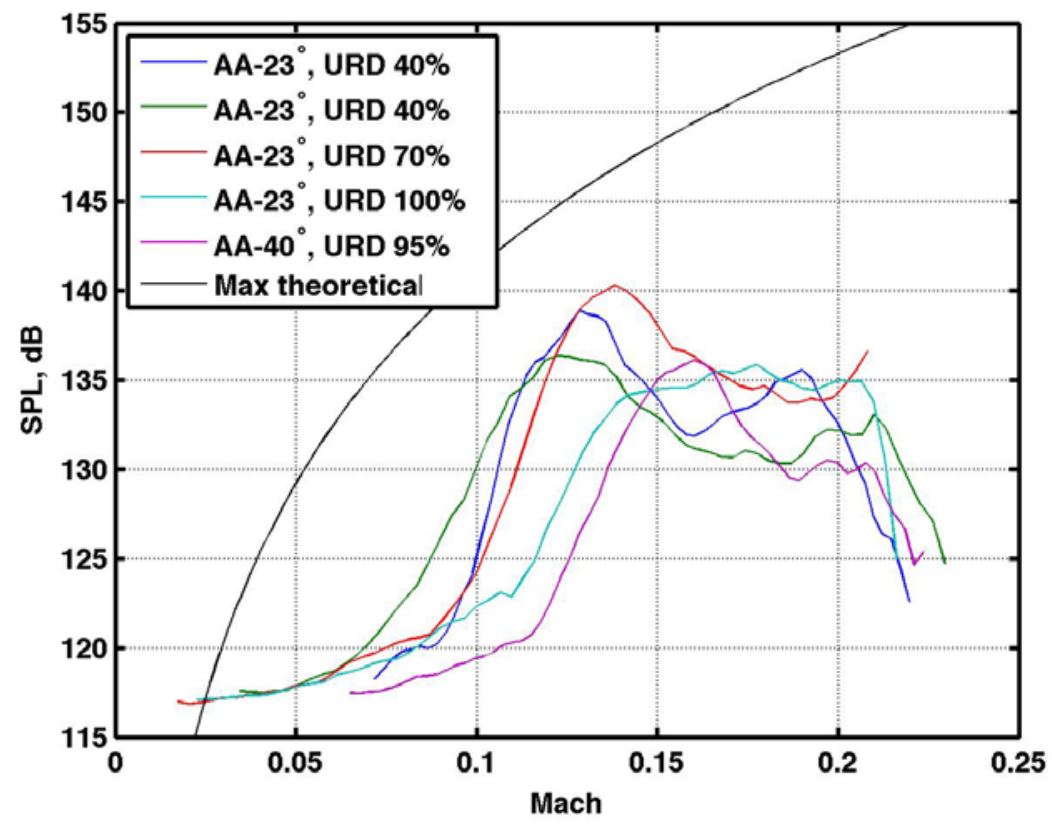

Figure 28. Sound pressure level versus Mach during open door landings for the cavity hot spot.

No structural response was noted during any of the open-door landings and the pilots reported no effects on the handling qualities of the airplane. Based on the results from the unplanned open-door landings, it was determined that no planned open-door landings were necessary because the pressure variations during resonance are not able to increase significantly over what had already been observed, due to the limited amount of flow energy available at low speeds, and the phenomenon did not pose a danger to the aircraft structure or the observatory systems.

\section{Additional Observations of Interest}

The AA was designed as a shear layer control to reduce adverse acoustic effects within the cavity. To quantify the effectiveness of the AA, the RMS of the pressure values within the cavity was normalized by the free stream dynamic pressure, as shown in Eq. (6):

$$
\text { aperture effectiveness }=\frac{p_{R M S}}{\bar{q}}
$$

Figure 29 shows a comparison between the 7-percent-scale wind-tunnel tests for the final configuration and actual flight data. The moving average, shown in the figure as a blue line, illustrates very good agreement with the wind tunnel data. 


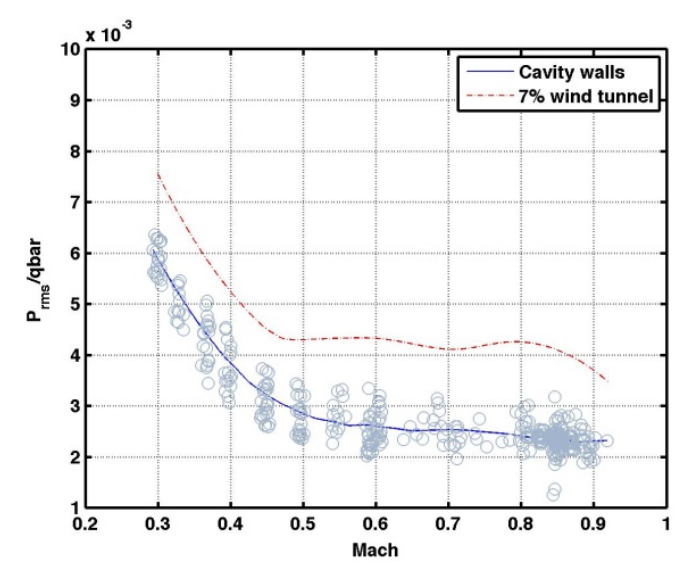

(a)

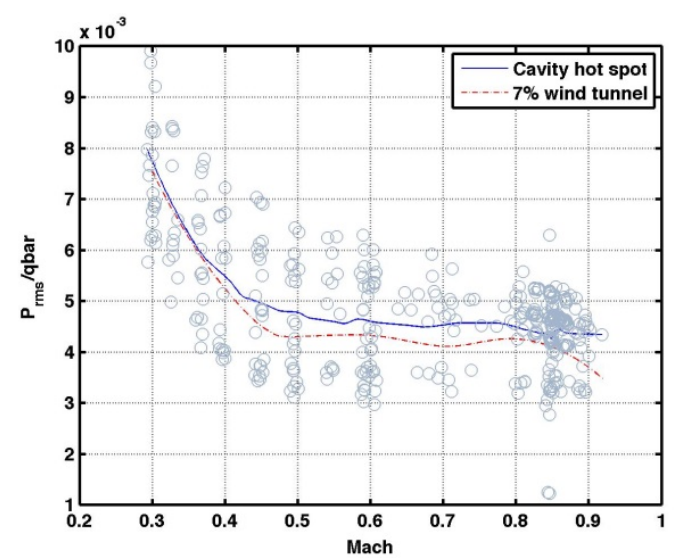

(b)

Figure 29. Aperture effectiveness (a) entire cavity. (b) cavity hot spot.

\section{B. Stability and Control}

As mentioned above, stability and control parameter estimation was performed in order to determine whether the modifications to the SOFIA airplane affected its aerodynamic characteristics. Parameter estimation maneuvers were performed throughout the flight envelope and at a wide range of AA/TA elevations and URD positions. In general, the SOFIA modifications were found to not have a substantial effect on the stability and control characteristics of the airplane. A few parameters showed changes between different flight segments and airplane configurations. Scatter in the parameter estimates made it difficult to discern if trends were present.

Some of the parameter estimation results are highlighted below. When plotted, the results from each maneuver set have been combined using a weighted average based on their Cramér-Rao bounds. Each point is accompanied by asymmetric error bounds that indicate the scatter in the set for that maneuver set, corresponding roughly to a 95-percent confidence interval in each direction. The confidence intervals are derived from the scatter in the results and the Cramér-Rao bounds of each original parameter estimate. Comparison data in the plots are shown as trend lines and 95-percent confidence regions that come from the same blending process as the symbols.

The reference area, span, and chord of the airplane are $5500 \mathrm{ft}^{2}, 195.68 \mathrm{ft}$, and $27.31 \mathrm{ft}$, respectively. The moment reference point of the airplane was fuselage station 1339.92 and water line 199.8.

\section{Closed-Door Results}

Estimates of $C_{n_{\beta}}$ are shown in Fig. 30, with different symbols corresponding to different flap deflections. Baseline data trends are separated by flap deflection, as well. The closed-door $C_{n_{\beta}}$ estimates had significant scatter, particularly at low speeds, as indicated by the large error bars. Below Mach 0.7, the estimates fell predominantly within the baseline confidence region, though the flap trends did not match. The Segment 2 closed-door points between Mach 0.4 and Mach 0.5 were considerably lower than the baseline data. At high speeds, the closed-door results were fairly consistent with each other and trended lower than the baseline data, suggesting a potential reduction in $C_{n_{\beta}}$ due to the modifications to the airplane. 


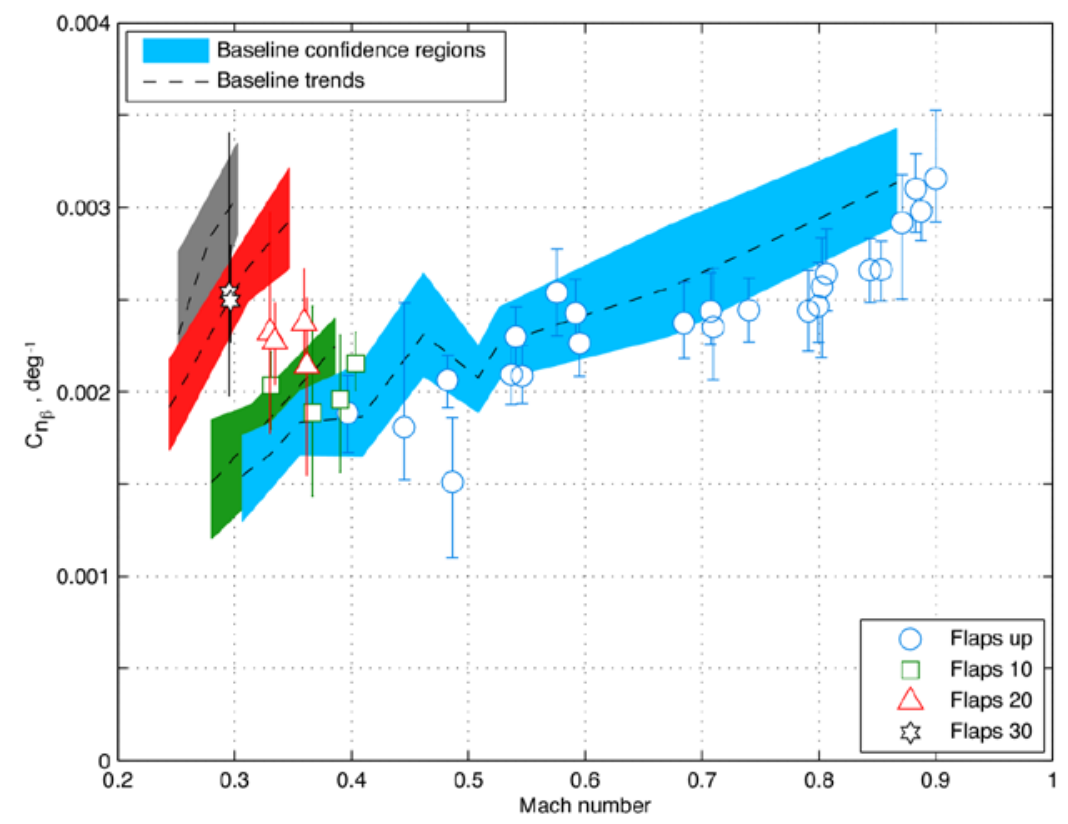

Figure 30. Estimates of $C_{n_{\beta}}$ for closed-door configuration.

Figure 31 shows estimates of the rudder effectiveness derivative, $C_{n_{\delta r}}$. At low and high speeds, the closed-door results agreed well with the baseline data. In the middle of the speed range, the baseline data showed a reduction in the magnitude of $C_{n_{\delta x}}$ that the closed-door results did not show. The closed-door data had a relatively well-behaved Mach number trend. It was concluded that the external modifications to the vehicle did not reduce the effectiveness of the rudder.

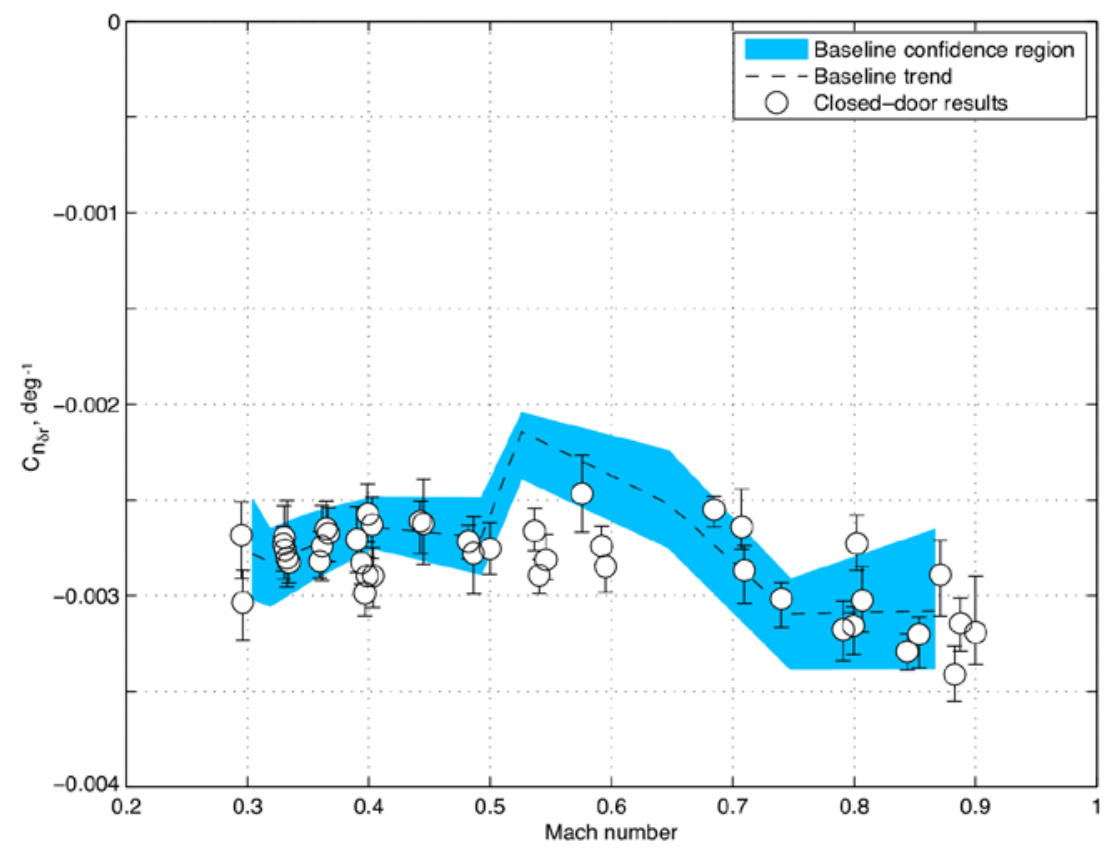

Figure 31. Estimates of $C_{n_{\delta r}}$ for closed-door configuration.

American Institute of Aeronautics and Astronautics 


\section{Open-Door Results}

Open-door estimates of $C_{n_{\beta}}$ are shown in Fig. 32. In general, the open-door results agree with the closed-door

confidence region, but the scatter associated with the open-door points is higher. Scatter was particularly high at higher Mach numbers, not only for different vehicle configurations, but also among the set of maneuvers at each test point, as shown by the error bars. It is difficult to conclude whether this is due to open-door aerodynamic changes, or related to differences in the styles of the maneuvers performed in Segment 2 versus those used in Segment 1. The Segment 2 inputs were generally larger in magnitude and shorter in duration than those of Segment 1 . The scatter also makes it hard to tell if there are any patterns in the results with respect to URD position and AA elevation; there do not appear to be any consistent patterns. The open-door $C_{n_{\beta}}$ estimates tended to be lower than the closed-door estimates, indicating a slight reduction in directional static stability, in addition to the reduction seen when going from the baseline airplane to the closed-door SOFIA configuration.

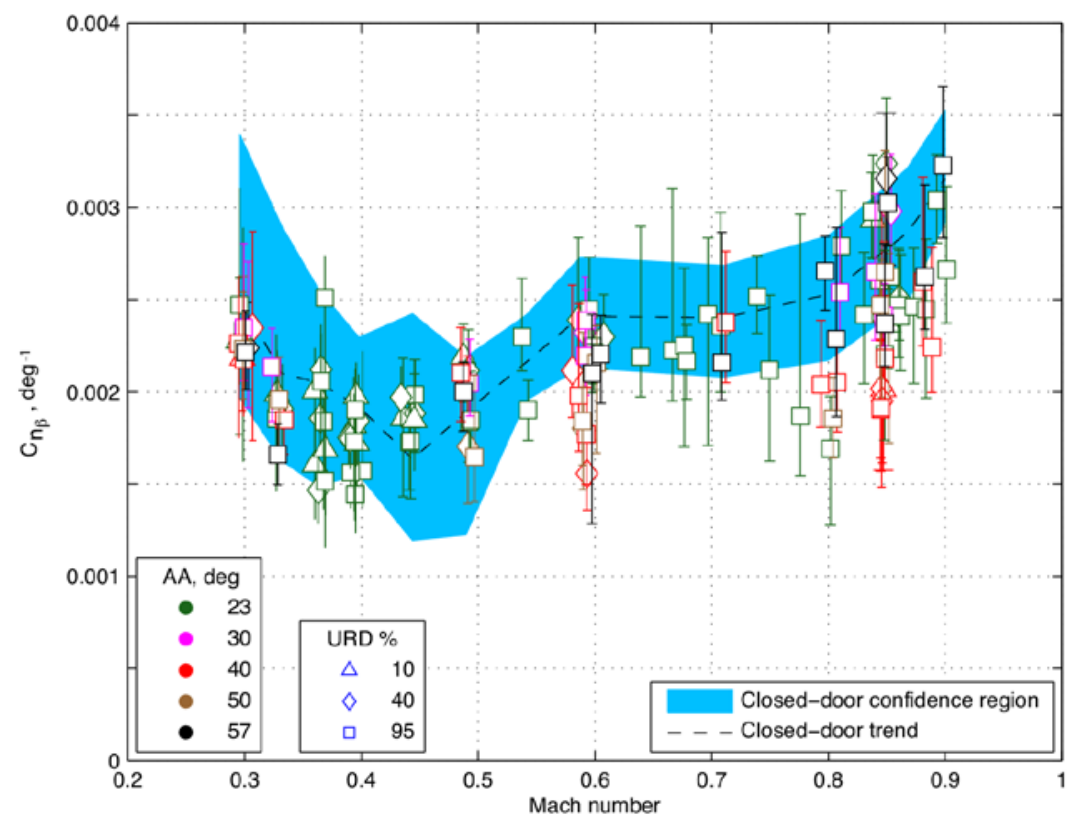

Figure 32. Estimates of $C_{n_{\beta}}$ for open-door configurations.

Figure 33 shows the $C_{n_{\tilde{\phi}}}$ estimates for the open-door configurations. Again, there was more scatter in the open-door results than for the closed-door. The open-door estimates, however, generally agreed well with the closed-door estimates. Some of the scatter at high speeds moved the estimates to an area of lower effectiveness than the closed-door data. Overall, there did not seem to be a significant loss of rudder effectiveness due to having the cavity door open. 




Figure 33. Estimates of $C_{n_{\delta r}}$ for open-door configurations.

Throughout the open-door flight tests, the pilots were generally unable to discern a change in handling qualities between flight with the cavity door open and flight with the cavity door closed. This reinforced the conclusion that, while there were some changes as a result of the modification, they were not significant.

\section{Conclusion}

The Stratospheric Observatory for Infrared Astronomy (SOFIA) is an airborne astronomical observatory developed through a partnership between the National Aeronautics and Space Administration and Deutsches Zentrum fur Luft- und Raumfahrt (DLR) (Cologne, Germany). The SOFIA airplane is a modified 747SP airplane (The Boeing Company, Chicago, Illinois) carrying an optical/infrared/sub-millimeter reflecting telescope with an effective diameter of $2.5 \mathrm{~m}$. The observatory was designed to perform observations between the altitudes of $35,000 \mathrm{ft}$ and $45,000 \mathrm{ft}$, where it is above 99 percent of the water vapor in the atmosphere.

The SOFIA airplane was modified for use as an observatory by installing the telescope and associated equipment in the aft section of the airplane. To provide maximum optical performance, an open-air cavity was created, and a door system was installed. To clear the airplane, and the observatory as a whole for its mission, a substantial flight envelope expansion process was necessary. The structural integrity of the modified airplane had to be verified. The acoustic environment inside the cavity had to be characterized to ensure that acoustic levels remained below design limits, both to prevent structural fatigue and to allow the observatory to meet image quality requirements. The stability and controllability of the modified airplane also had to be evaluated, both in closed-door and open-door configurations. Functionality of the production pitot-static system of the airplane had to be verified, and the research air data system had to be calibrated. In addition, all of the new systems installed to support the telescope and the observatory had to be tested and their functionality verified.

Three flight test segments were performed. Segment 0 consisted of envelope clearance for a ferry flight to NASA Dryden Flight Research Center (Edwards, California). Segment 1 consisted of tests performed to clear the closed-door envelope. Segment 2 consisted of open-door envelope clearance and checkout of various observatory systems. Major concerns during these flight segments included acoustic resonance during open-door operations, possible effects on the stability and control of the airplane, and possible effects on air data systems.

To address acoustic concerns, the cavity was thoroughly instrumented with pressure sensors. Analysis performed on the data from these sensors identified overall acoustic levels in the cavity as well as frequency content from individual sensors. There were no significant cavity tones at scientific observation flight conditions; the only substantial cavity tone was found during open-door landings. This tone, however, does not excite the cavity structure 
or TA components. During sideslip maneuvers at certain flight conditions, the SPL rose above set limits, but the frequency content was broadband in nature, and posed no danger to any airplane structures. The cavity behavior compared favorably with the 7-percent-scale wind-tunnel results. In fact, the actual measured cavity acoustics were even more benign than the 7-percent-scale wind-tunnel results suggested.

The stability and control characteristics of the 747SP airplane were not significantly affected by the SOFIA modifications, regardless of configuration. There was, however, a general increase in data scatter for results from the open-door configuration flights, compared to the closed-door and baseline results. It is thought that at least some of the increased scatter was due to maneuver technique. There was a possible reduction in $C_{n_{\beta}}$ in the closed-door configuration, when compared to baseline results, and in the open-door configuration, when compared with the closed-door results.

In conclusion, from an aerodynamics and acoustics standpoint, the performance of the SOFIA airplane was better than predicted. Neither the air data systems of the SOFIA airplane, nor the research flush air data sensing system were affected by the SOFIA modifications. There are no outstanding aerodynamics or acoustics problems, and the airplane has been cleared, from an aerodynamics and acoustics perspective, for full-envelope science operations.

\section{References}

${ }^{1}$ Thronson, H. A., Jr. and Erickson, E. F., Airborne Astronomy Symposium, NASA/CP-1984-2353, 1984.

${ }^{2}$ Rose, W. C., SOFIA TA Cavity Aerodynamics: Wind Tunnel to Flight, NASA CR, TBD, TBD.

${ }^{3}$ Knuz, N., "The SOFIA Aircraft and its Modification,” Proceedings of SPIE Vol. 4857, 2003.

${ }^{4}$ Kehoe, M. W. etc., Stratospheric Observatory for Infrared Astronomy (SOFIA) Acoustical Resonance Technical Assessment Report, NASA/TM-2009-215730, 2009.

${ }^{5}$ Srinivasan, G. R., “Flowfield and Acoustic Characteristics of Telescope Cavity in SOFIA Platform,” AIAA-95-1862, 1995.

${ }^{6}$ Klotz, S. P., “Numerical Simulation of the SOFIA Flow Field,” NASA/CR-197757, 1995.

${ }^{7}$ Welch, P.D., "The Use of Fast Fourier Transform for the Estimation of Power Spectra: A Method Based on Time Averaging Over Short, Modified Periodograms,” IEEE Transactions on Audio Electroacoustics, AU-15, 70-73, 1967.

${ }^{8}$ Maine, R. E. and Iliff, K. W., "Application of Parameter Estimation to Aircraft Stability and Control, The Output Error Approach,” NASA/RP-1168, 1986.

${ }^{9}$ Morelli, E. A., "Practical Aspects of the Equation-Error Method for Parameter Estimation,” AIAA-2006-6144, 2006.

${ }^{10}$ Murray, J. E., and R. E. Maine, pEst Version 2.1 User's Manual, NASA/TM-1987-88280, 1987.

${ }^{11}$ Haering, E. A., Jr., “Airdata Calibration of a High-Performance Aircraft for Measuring Atmospheric Wind Profiles," NASA/TM-1990-101714, 1990.

${ }^{12}$ Whitmore, S. A., Cobleigh, B. R., and Haering, E.A., "Design and Calibration of the X-33 Flush Airdata Sensing (FADS) System,” NASA/TM-1998-206540, 1998.

${ }^{13}$ Rossiter, J. E., "Wind Tunnel Experiments on the Flow over Rectangular Cavities at Subsonic and Transonic Speeds," Aeronautical Research Council Reports and Memo No. 3438, 1964. 